\title{
VISCOSITY SOLUTIONS OF THE BELLMAN EQUATION FOR EXIT TIME OPTIMAL CONTROL PROBLEMS WITH NON-LIPSCHITZ DYNAMICS *,**
}

\author{
Michael MALisofF ${ }^{1}$
}

\begin{abstract}
We study the Bellman equation for undiscounted exit time optimal control problems with fully nonlinear Lagrangians and fully nonlinear dynamics using the dynamic programming approach. We allow problems whose non-Lipschitz dynamics admit more than one solution trajectory for some choices of open loop controls and initial positions. We prove a uniqueness theorem which characterizes the value functions of these problems as the unique viscosity solutions of the corresponding Bellman equations that satisfy appropriate boundary conditions. We deduce that the value function for Sussmann's Reflected Brachystochrone Problem for an arbitrary singleton target is the unique viscosity solution of the corresponding Bellman equation in the class of functions which are continuous in the plane, null at the target, and bounded below. Our results also apply to degenerate eikonal equations, and to problems whose targets can be unbounded and whose Lagrangians vanish for some points in the state space which are outside the target, including Fuller's Example.
\end{abstract}

Mathematics Subject Classification. 49L25, 93Cxx.

Received April 19, 2000. Revised December 22, 2000.

\section{INTRODUCTION}

This paper continues our work ( $c f$. [14]) on uniqueness questions for viscosity solutions of Hamilton-JacobiBellman equations (HJBE's) arising from deterministic optimal control problems with exit times (cf. [2]). We prove a general uniqueness theorem characterizing the value functions for problems of this type for fully nonlinear systems as the unique viscosity solutions of the corresponding HJBE's that satisfy appropriate boundary conditions. We allow problems whose dynamical laws admit more than one solution trajectory for some choices of open loop controls and initial positions. The class of problems includes Sussmann's Reflected Brachystochrone Problem (RBP) (cf. [12,21,22], and Sect. 5), eikonal equations (cf. [2,18], and Sects. 6.1, 6.2), and problems with unbounded targets and unbounded Lagrangians $\ell$ for which $\ell(\cdot, a)$ is zero at some points outside the target for some choices of the input $a$. As a special case, we show that the RBP value function is the unique viscosity

Keywords and phrases: Viscosity solutions, dynamical systems, Reflected Brachystochrone Problem.

* This research was supported in part by NSF Grant DMS95-00798. This paper was revised on December 20, 2000.

** This work was done while the author was a University and Louis Bevier Graduate Fellow in the Department of Mathematics at Rutgers University. It is part of the author's dissertation under the direction of Professor Héctor J. Sussmann. Some of this work was presented during the session "Optimal Control and Optimization" of the 38 ${ }^{\text {th }}$ IEEE Conference on Decision and Control in Phoenix, Arizona on December 7, 1999 and published in preliminary form in the conference proceedings.

1 Department of Systems Science and Mathematics, Washington University in Saint Louis, One Brookings Drive, Campus Box 1040, Saint Louis, MO 63130-4899, U.S.A.; e-mail: malisoff@zach.wustl.edu 
solution of the corresponding HJBE in the class of continuous functions which vanish on the target and which are bounded below.

Value function characterizations of this kind have been studied by many authors for a large number of stochastic and deterministic optimal control problems and for dynamic games. The characterizations have been applied to the convergence of numerical schemes for approximating optimal control value functions and differential game values with error estimates, singular perturbation problems, asymptotics problems, $H^{\infty}$-control, and much more. See for example the books [2] and [9] and the hundreds of references cited therein. For surveys of numerical analysis applications of viscosity solutions, see [4] and [19], and for uniqueness characterizations for the HJBE for discounted exit time problems, see [2]. For uniqueness characterizations for general Hamilton-Jacobi equations which do not necessarily arise as Bellman equations for control problems, see for example [1] and [7]. However, these earlier characterizations cannot be applied to many standard exit time problems whose dynamics are non-Lipschitz or whose Lagrangians vanish for some points outside the target. In fact, one easily finds exit time problems for which the Lagrangian vanishes for some points outside the target and for which the corresponding HJBE has more than one proper viscosity solution. For example, consider the dynamics $\dot{x}(t)=u(t) \in[-1,1]$, and choose the Lagrangian $\ell(x, a)=(x+2)^{2}(x-2)^{2} x^{2}(x+1)^{2}(x-1)^{2}$. Let $v_{1}$ and $v_{2}$ denote the value functions for the exit time problem of bringing points to the targets $\mathcal{T}_{1}=\{0\}$ and $\mathcal{T}_{2}=\{0,2,-2\}$, respectively, using the dynamics $\dot{x}=u \in[-1,+1]$ and the running cost $\ell$ (cf. (2.2) for the problem formulation). One can easily check that $v_{1}$ and $v_{2}$ are both proper viscosity solutions of the associated HJBE on $\mathbb{R} \backslash \mathcal{T}$ with the target $\mathcal{T}:=\mathcal{T}_{1}$ ( $c f$. (2.3) below). One checks that with the target choice $\mathcal{T}:=\mathcal{T}_{1}$, the problem satisfies all hypotheses of the well-known theorems which characterize value functions of exit time control problems as the unique proper viscosity solutions of $(2.3)$ which are zero on $\mathcal{T}$ except that the positive lower bound requirement on $\ell$ is not satisfied ( $c f .[2,5]$, and [15]).

Our work is part of a larger research program which extends uniqueness results from viscosity theory to versions covering well-known optimal control problems with unbounded cost functionals or dynamics that do not have uniqueness of solutions. For results on the Bellman equation for infinite horizon problems with non-Lipschitz dynamics, see [11]. For uniqueness characterizations for the Bellman equation for certain linearquadratic problems, see [3] (which covers finite horizon cases) and [8] (which covers the infinite horizon case). A uniqueness characterization for a large class of exit time problems whose dynamics are Lipschitz but whose Lagrangians vanish at some points outside the target is in [14], which gives a uniqueness characterization for the Fuller Problem (FP) as a special case ( $c f$. Sect. 6, the book [25], which is devoted entirely to variants of the Fuller Problem, and [23]).

This paper is organized as follows. In Section 2, we introduce the notions of Lipschitz upper envelopes and coercive transience and some notation, and we state our first main result. Loosely speaking, a Lipschitz upper envelope is a dynamical law $f$ which can be closely approximated by Lipschitz dynamical laws which admit at least as many trajectories as $f$ does (cf. Def. 2.2). A coercively transient control problem is one for which the dynamics is Lipschitz outside a thin set ( $c f$. Def. 2.3). We also review the definitions of viscosity solutions and relaxed controls. In Section 3, we state lemmas from the theory of viscosity solutions and relaxed controls. We prove our first main result in Section 4. In Section 5, we use the main result to deduce the uniqueness characterization for the RBP. In Section 6, we give three variants of the main result which apply to cases where the hypotheses of the main result are not satisfied. The third of these variants applies to problems with non-Lipschitz dynamics and Lagrangians which vanish for some points outside the target (cf. Sect. 6.3). This third variant is based on a generalization of a result from [14] which we prove in the appendix, and it gives the uniqueness characterizations for the Fuller Problem from [14] (e.g., Cor. 6.5 below) as special cases. In particular, we show that the Fuller Problem value function is the unique viscosity solution of the corresponding HJBE in a class of functions which includes functions which are not bounded below. We also deduce free boundary uniqueness characterizations for degenerate eikonal equations for which the speed of the medium is non-Lipschitz ( $c f$. Ex. 6.1 and Ex. 6.2). For a very different treatment of the HJBE for exit time problems with Lipschitz dynamics based on asymptotical properties of trajectories which shows that the FP value function is the unique viscosity solution of its HJBE in a suitable class of nonnegative functions, see [17]. 


\section{Notation, DEFinitions, And StATEMEnt of FiRst main RESUlt}

Let $A$ be a compact normed vector space, and let $f: \mathbb{R}^{N} \times A \rightarrow \mathbb{R}^{N}$ be continuous. Let $\mathcal{A}$ denote the set of measurable functions $[0,+\infty) \rightarrow A$. This paper will consider optimal control problems with the dynamics $f$, and we will take $\mathcal{A}$ as the set of admissible controls. Recall that if $A$ is a compact set, then a continuous dynamical law $h: \mathbb{R}^{N} \times A \rightarrow \mathbb{R}^{N}$ for an optimal control problem is called Lipschitz on $S$ if $S \subseteq \mathbb{R}^{N}$ and if there exists a constant $L>0$ such that $\|h(x, a)-h(y, a)\| \leq L\|x-y\|$ for all $x, y \in S$ and all $a \in A$. Dynamical laws which are Lipschitz on $\mathbb{R}^{N}$ will be called Lipschitz. For all spaces $S_{1}$ and $S_{2}$, we let $C\left(S_{1}, S_{2}\right)$ denote the set of all continuous functions $h: S_{1} \rightarrow S_{2}$. The set of all functions in $C\left(\mathbb{R}^{N} \times A, \mathbb{R}^{N}\right)$ which are Lipschitz will be denoted by $C_{\text {lip }}\left(\mathbb{R}^{N} \times A, \mathbb{R}^{N}\right)$.

Since $A$ is a compact metric space, we can view our controls $\alpha \in \mathcal{A}$ as members of the larger class $\mathcal{A}^{r}$ of measure-valued relaxed controls on $A$ (cf. [2] and [24]). By this we mean the following. We define $\mathcal{A}^{r}:=\left\{\right.$ measurable functions $\left.[0, \infty) \rightarrow A^{r}\right\}$, where $A^{r}$ is the set of Radon probability measures on $A$ (i.e., the probability measures supported on $A$ whose domains include the smallest $\sigma$-algebra on $A$ containing all the open subsets of $A$ ). In what follows, we let $C(U)$ denote the set of continuous real-valued functions on $U$, and $C^{1}$ means continuous with one continuous derivative. We topologize $A^{r}$ as a subset of the dual of $C(A)$ with the topology of weak- $\star$ convergence. By identifying $\mathcal{A}$ with the set of Dirac probability measures on $A$ (i.e., probability measures that put weight one on a single point of $A$ at each time), we will view $\mathcal{A}$ as a subset of $\mathcal{A}^{r}$. By $\mathcal{A}^{r} \ni \alpha_{n} \rightarrow \bar{\alpha} \in \mathcal{A}^{r}$ weak- $\star$, we will mean that

$$
\int_{0}^{t} \int_{A}(g(s))(a) \mathrm{d}\left(\alpha_{n}(s)\right)(a) \mathrm{d} s \quad \rightarrow \quad \int_{0}^{t} \int_{A}(g(s))(a) \mathrm{d}(\bar{\alpha}(s))(a) \mathrm{d} s \quad \text { as } \quad n \rightarrow \infty
$$

for each Lebesgue integrable function $g:[0, t] \rightarrow C(A)$ and each $t>0$. For any continuous function $\Phi:$ $\mathbb{R}^{N} \times A \rightarrow \mathbb{R}^{M}$, we define $\Phi^{r}: \mathbb{R}^{N} \times A^{r} \rightarrow \mathbb{R}^{N}$ by $\Phi^{r}(x, m):=\int_{A} \Phi(x, a) \mathrm{d} m(a)$.

For our general continuous dynamics $f$, there may be points $x \in \mathbb{R}^{N}$ and controls $\alpha \in \mathcal{A}$ for which there are several trajectories of $\dot{y}=f(y, \alpha(s))$ starting at $x$ (i.e., absolutely continuous functions $\phi$ satisfying $\dot{\phi}=$ $f(\phi, \alpha(s))$ for a.a. $t \geq 0$ and $\phi(0)=x)$. This motivates the following definition. For $h: \mathbb{R}^{N} \times A \rightarrow \mathbb{R}^{N}$ continuous, $S \subseteq \mathbb{R}^{N}, \alpha \in \mathcal{A}$, and $x \in \mathbb{R}^{N}$, we set

$$
\operatorname{Traj}_{\alpha}(x, h, S):=\left\{\begin{array}{c}
\text { trajectories } \phi \text { of } \dot{y}(s)=h(y(s), \alpha(s)), y(0)=x \text { defined on } \\
{[0,+\infty) \text { for which there exists a } \bar{t}<\infty \text { such that } \phi(\bar{t}) \in S}
\end{array}\right\}
$$

and $\operatorname{Traj}(x, h, A, S)=\cup\left\{\operatorname{Traj}_{\beta}(x, h, S): \beta \in \mathcal{A}\right\}$. We always assume that $\operatorname{Traj}_{\alpha}\left(x, f, \mathbb{R}^{N}\right) \neq \emptyset$. Set $\mathbb{R}_{+}=$ $[0,+\infty)$. We let $C_{\text {inc }}\left(\mathbb{R}^{N} \times A, \mathbb{R}_{+}\right)$denote those continuous functions $h: \mathbb{R}^{N} \times A \rightarrow \mathbb{R}_{+}$with the property that for all $x \in \mathbb{R}^{N}$ and for all values $a, a^{\prime} \in A$ with $\|a\| \leq\left\|a^{\prime}\right\|$, we have $h(x, a) \leq h\left(x, a^{\prime}\right)$.

This paper will consider optimal control problems whose objectives are to find cost-minimizing paths which bring points to a fixed closed target set $\mathcal{T} \subseteq \mathbb{R}^{N}$. For any continuous function $h: \mathbb{R}^{N} \times A \rightarrow \mathbb{R}^{N}$, we let $\mathcal{R}_{h}$ denote the set of points $x \in \mathbb{R}^{N}$ for which $\operatorname{Traj}(x, h, A, \mathcal{T})$ is nonempty. We sometimes write $\mathcal{R}_{h}(A)$ to emphasize the control set, and $\mathcal{R}$ will denote $\mathcal{R}_{f}$ when this would not lead to confusion. We also define the function $T_{A, f}: \mathcal{R}_{f} \times \mathcal{R}_{f} \rightarrow[0,+\infty]$ by

$$
T_{A, f}(p, q):=\inf \{t \geq 0: \exists \phi \in \operatorname{Traj}(p, f, A,\{q\}) \text { s.t. } \phi(t)=q\} .
$$

We generally assume $T_{A, f}$ is continuous, i.e., $T_{A, f}$ is everywhere finite and $T_{A, f}: \mathcal{R}_{f} \times \mathcal{R}_{f} \rightarrow[0, \infty)$ is continuous (but see Sect. 6.3 for cases where $T_{A, f}$ is not continuous). For each $S \subset \mathbb{R}^{N}$ and $\phi \in \operatorname{Traj}(x, f, A$, $S$ ), we set $\tau_{S}(\phi)=\inf \{t \geq 0: \phi(s) \in S\}$. When $S$ is a singleton $\{p\}$, we write $\tau_{p}$ instead of $\tau_{\{p\}}$. We write $\tau(\phi)$ instead of $\tau_{\mathcal{T}}(\phi)$, and we call $\tau(\phi)$ the exit time of $\phi$. Fix $\ell \in C_{\text {inc }}\left(\mathbb{R}^{N} \times A, \mathbb{R}_{+}\right)$. This paper considers the following 
problem:

$$
\text { For each } x \in \mathcal{R}_{f}(A) \text {, infimize } \int_{0}^{\tau(\phi)} \ell(\phi(s), \alpha(s)) \mathrm{d} s \text { over all } \phi \in \operatorname{Traj}_{\alpha}(x, f, \mathcal{T}) \text { and } \alpha \in \mathcal{A} .
$$

We generally consider cases where the Lagrangian $\ell$ is bounded below by a positive constant, in which case the set $\operatorname{Traj}_{\alpha}(x, f, \mathcal{T})$ in $(2.2)$ can be replaced by $\operatorname{Traj}_{\alpha}\left(x, f, \mathbb{R}^{N}\right)$ (but see Sect. 6.3 for results for cases where $\ell$ vanishes for some points outside $\mathcal{T}$ ). The value function of $(2.2)$ will be denoted by $v_{f, \ell, A}$. More generally,

$$
v_{h, \ell, A}^{S}(x):=\inf \left\{\int_{0}^{\tau_{S}(\phi)} \ell(\phi(s), \alpha(s)) \mathrm{d} s: \alpha \in \mathcal{A}, \phi \in \operatorname{Traj}_{\alpha}(x, h, S)\right\}
$$

for continuous functions $h: \mathbb{R}^{N} \times A \rightarrow \mathbb{R}^{N}, S \subseteq \mathbb{R}^{N}$, and $x \in \mathbb{R}^{N}$. When $S$ is a singleton $\{q\}$, we write $v_{h, \ell, A}^{q}$ instead of $v_{h, \ell, A}^{\{q\}}$, and we write $v_{f, \ell, A}$ instead of $v_{f, \ell, A}^{\mathcal{T}}$. We also set

$$
v_{\infty}(x)=\inf \left\{\int_{0}^{\infty} \ell(\phi(s), \alpha(s)) \mathrm{d} s: \alpha \in \mathcal{A}, \phi \in \operatorname{Traj}_{\alpha}\left(x, f, \mathbb{R}^{N}\right)\right\} .
$$

Define the Hamiltonian $H: \mathbb{R}^{N} \times A \rightarrow \mathbb{R}$ by

$$
H(x, p):=\max _{a \in A}\{-f(x, a) \cdot p-\ell(x, a)\}
$$

and set $\mathcal{M}(p, x)=\left\{\bar{a} \in A:-f(x, \bar{a}) \cdot p-\ell(x, \bar{a})=\max _{a \in A}[-f(x, a) \cdot p-\ell(x, a)]\right\}$ for all $x, p \in \mathbb{R}^{N}$. The Hamilton-Jacobi-Bellman equation (HJBE) for our problem (2.2) can then be expressed as

$$
H(x, D v(x))=0 \quad \text { on } \quad \mathcal{R}_{f}(A) \backslash \mathcal{T} .
$$

We give conditions under which $v_{f, \ell, A}$ is the unique solution of (2.3) that satisfies appropriate boundary conditions. By solution, we mean the following:

Definition 2.1. Let $\mathcal{G} \subseteq \mathbb{R}^{N}$ be open, let $S \supseteq \mathcal{G}$, and let $F: \mathbb{R}^{N} \times \mathbb{R}^{N} \rightarrow \mathbb{R}$ and $w: S \rightarrow \mathbb{R}$ be continuous. We call $w$ a (viscosity) solution of $F(x, D w(x))=0$ on $\mathcal{G}$ if the following conditions hold:

$\left(V_{1}\right)$ If $\gamma: \mathcal{G} \rightarrow \mathbb{R}$ is $C^{1}$ and $x_{o}$ is a local minimum point of $w-\gamma$, then $F\left(x_{o}, D \gamma\left(x_{o}\right)\right) \geq 0$.

$\left(V_{2}\right)$ If $\lambda: \mathcal{G} \rightarrow \mathbb{R}$ is $C^{1}$ and $x_{1}$ is a local maximum point of $w-\lambda$, then $F\left(x_{1}, D \lambda\left(x_{1}\right)\right) \leq 0$.

We call $w$ a viscosity supersolution (resp, subsolution) of $F(x, D w(x))=0$ on $\mathcal{G}$ if condition $\left(V_{1}\right)$ (resp., $\left.\left(V_{2}\right)\right)$ holds.

We also use the following equivalent definition of viscosity solutions based on the superdifferentials $D^{+} w(x)$ and subdifferentials $D^{-} w(x)$ of $w$. Let $\mathcal{G}, S, F$, and $w$ be as in Definition 2.1, and define

$$
\begin{aligned}
& D^{+} w(x):=\left\{p \in \mathbb{R}^{N}: \limsup _{\mathcal{G} \ni y \rightarrow x} \frac{w(y)-w(x)-p \cdot(y-x)}{\|x-y\|} \leq 0\right\} \\
& D^{-} w(x):=\left\{p \in \mathbb{R}^{N}: \liminf _{\mathcal{G} \ni y \rightarrow x} \frac{w(y)-w(x)-p \cdot(y-x)}{\|x-y\|} \geq 0\right\} .
\end{aligned}
$$

One checks that condition $\left(V_{1}\right)$ is equivalent to the condition

$\left(V_{1}^{\prime}\right) \quad F(x, p) \geq 0$ for all $x \in \mathcal{G}$ and $p \in D^{-} w(x)$ 
and that condition $\left(V_{2}\right)$ is equivalent to the condition

$\left(V_{2}^{\prime}\right) \quad F(x, p) \leq 0$ for all $x \in \mathcal{G}$ and $p \in D^{+} w(x)$

so we equivalently define viscosity solutions by saying that $w$ is a viscosity solution of $F(x, D w(x))=0$ on $\mathcal{G}$ exactly when $\left(V_{1}^{\prime}, V_{2}^{\prime}\right)$ hold.

We also say that $w$ is the complete (viscosity) solution of $F(x, D w(x))=0$ on $\mathcal{G}$ in a class $\mathcal{F}$ of functions in $C(\mathcal{G})$ if $w \in \mathcal{F}$, if $\left(V_{1}, V_{2}\right)$ hold, and if $w$ is the maximal subsolution and minimal supersolution of this equation in $\mathcal{F}$, i.e., if $\tilde{w} \in \mathcal{F}$ is a viscosity supersolution (resp., subsolution) of $F(x, D \tilde{w}(x))=0$ on $\mathcal{G}$, then $w(x) \leq \tilde{w}(x)$ (resp., $w(x) \geq \tilde{w}(x)$ ) for all $x \in \mathcal{G}$. We begin by studying viscosity solutions of the Bellman equation (2.3) satisfying the side conditions

$$
\left\{\lim _{\mathcal{R}_{f} \ni x \rightarrow x_{0}} v(x)=+\infty \text { for all } x_{o} \in \partial\left(\mathcal{R}_{f}\right), v \equiv 0 \text { on } \mathcal{T} \text {, and } v \text { is bounded below }\right\}
$$

Note that the limit condition in (SC) holds vacuously for cases where $\mathcal{R}_{f}=\mathbb{R}^{N}$. For results on viscosity solutions of (2.3) which are not bounded below, see Section 6.3. In much of what follows, we will be studying cases where the dynamics $f$ of (2.2) is an "upper envelope" of Lipschitz dynamics, in the following sense (but see Rem. 2.5, Rem. 2.6, and Sect. 6 for related results which hold under much weaker hypotheses):

Definition 2.2. We call $f$ a Lipschitz upper envelope if there exists a sequence $f_{n} \in C_{\text {lip }}\left(\mathbb{R}^{N} \times A, \mathbb{R}^{N}\right)$ such that the following conditions hold:

1. $\mathcal{R}:=\mathcal{R}_{f} \equiv \mathcal{R}_{f_{n}}$ is open, and $f_{n} \rightarrow f$ uniformly on compact sets.

2. $\operatorname{Traj}\left(x, f_{n}, A, \mathcal{T}\right) \subseteq \operatorname{Traj}\left(x, f_{1}, A, \mathcal{T}\right)$ for each $n \in \mathbb{N}$ and $x \in \mathcal{R}$.

3. For each $p \in \mathbb{N}, x \in \mathcal{R}$, and $\phi$ in $\operatorname{Traj}_{\alpha}\left(x, f, \mathbb{R}^{N}\right)$, there is a $\beta \in \mathcal{A}$ so that $\phi \in \operatorname{Traj}_{\beta}\left(x, f_{p}, \mathbb{R}^{N}\right)$ and so that $\|\alpha\| \geq\|\beta\|$ a.e.

4. For each $x \in \mathcal{R}$ and $p \in \mathbb{R}^{N}$, there is an $\bar{a} \in \mathcal{M}(p, x)$ such that $f_{n}(x, \bar{a}) \cdot p \leq f(x, \bar{a}) \cdot p$ for all $n$.

Roughly speaking, a Lipschitz upper envelope is therefore a dynamical law which is closely approximated from below by Lipschitz dynamical laws with "at least as many" trajectories. For example,

$$
f\left((x, y)^{\prime},(a, b, c, d)^{\prime}\right)=\left(\beta_{1} k_{1}(a)|x|^{\delta_{1}}+\beta_{2} k_{2}(b)|y|^{\delta_{2}}, \beta_{3} k_{3}(c)|x|^{\delta_{3}}+\beta_{4} k_{4}(d)|y|^{\delta_{4}}\right)^{\prime}
$$

is a Lipschitz upper envelope on $\mathbb{R}^{2} \times[-1,+1]^{4}$ for any $\delta_{j} \in(0,1), \beta_{j} \geq 0$, and strictly increasing, odd, surjective functions $k_{j} \in C([-1,+1],[-1,+1])$ (cf. Sect. 5). If $f$ is a Lipschitz upper envelope, and if $\{f(x, a) \times \ell(x, a)$ : $a \in A\}$ is convex for all $x$, then we call $f$ a convex Lipschitz upper envelope (colue). In that case, we sometimes refer to $(f, \ell)$ as a colue to emphasize the Lagrangian. Notice that relaxed trajectories of colues can be realized as trajectories without changing the running costs ( $c f$. Lem. 3.8). Condition 4 of Definition 2.2 is called the nonexpansiveness condition. If $f$ satisfies all requirements for being a colue except Condition 4 , then we call it an expansive colue. For uniqueness characterizations for expansive colues, see Section 6.2.

Set $U L(f)=\cup\left\{S \subseteq \mathbb{R}^{N}: f\lceil[S \times A]\right.$ is Lipschitz on $S\}$. We study cases where $f$ has a non-Lipschitz set $\mathbb{R}^{N} \backslash U L(f)$ which is, in a suitable sense, thin (but see Sect. 6.1 for results where the thinness condition we now give is not satisfied). For $\ell \equiv 1$, thinness roughly means that those trajectories starting outside $U L(f)$ can be replaced by trajectories that immediately enter $U L(f)$ without increasing the travel time. In that case, thinness means it is always advantageous to exit into $U L(f)$ from initial points outside $U L(f) \cup \mathcal{T}$. More generally, we use the following thinness notion:

Definition 2.3. For each $x \in \mathcal{R} \backslash \mathcal{T}$ and $\alpha \in \mathcal{A}$, let $\operatorname{Traj}_{\alpha}^{c o}(x, f, \mathcal{T})$ denote those $\phi \in \operatorname{Traj}_{\alpha}(x, f, \mathcal{T})$ that admit $t, t^{\prime} \in(0, \tau(\phi)), \beta \in \mathcal{A}, \psi \in \operatorname{Traj}_{\beta}(x, f, \mathcal{T}), N \in \mathbb{N}$, and open sets $\left\{S_{n}\right\}_{n=N}^{\infty}$ in $\mathbb{R}^{N}$ such that $\phi(t)=\psi\left(t^{\prime}\right)$, 
$S_{n} \supseteq$ Trace $\psi\left\lceil\left[1 / n, t^{\prime}\right]\right.$ and $f$ is Lipschitz on $S_{n}$ for all $n \geq N$, and

$$
\int_{0}^{t} \ell(\phi(s), \alpha(s)) \mathrm{d} s \geq \int_{0}^{t^{\prime}} \ell(\psi(s), \beta(s)) \mathrm{d} s .
$$

For $x \in \mathcal{T}$, we set $\operatorname{Traj}_{\alpha}^{c o}(x, f, \mathcal{T}) \equiv \operatorname{Traj}_{\alpha}(x, f, \mathcal{T})$. We call $\bigcup_{\alpha \in \mathcal{A}} \operatorname{Traj}_{\alpha}^{c o}(x, f, \mathcal{T})$ the coercifiable trajectories at $x$ (relative to $f, \mathcal{T}$, and $\ell$ ). We call a pair $(f, \ell)$ (coercively) transient (relative to $A$ ) if for all $x \in \mathcal{R}$, each $\phi \in \operatorname{Traj}(x, f, A, \mathcal{T})$ is a coercifiable trajectory at $x$.

We will begin by proving the following theorem:

Theorem 2.1. Let $A$ be a compact normed vector space, $\mathcal{T} \subset \mathbb{R}^{N}$ be closed, $(f, \ell)$ be a transient colue, and $T_{A, f}$ be continuous. Assume $\ell$ is bounded below by a positive constant. Then $v_{f, \ell, A}$ is the unique viscosity solution of (2.3) in $C(\mathcal{R})$ which satisfies the side conditions (SC).

Following [8], we then show how the hypotheses of the theorem can be relaxed if $v_{f, \ell, A}$ has locally bounded superdifferentials or subdifferentials (cf. Sect. 6). Roughly speaking, Theorem 2.1 is true if $(f, \ell)$ is an expansive colue as long as we assume that $v_{f, \ell, A}$ has locally bounded subdifferentials and the other hypotheses are kept the same (cf. Sect. 6.2). By assuming that $v_{f, \ell, A}$ has locally bounded superdifferentials, we extend the result to cover data which are not coercively transient ( $c f$. Sect. 6.1). In either case, we show that $v_{f, \ell, A}$ is the unique viscosity solution of $(2.3)$ in a class of functions in $C(\mathcal{R})$ each of whose members has locally bounded sub- or superdifferentials. We also cover cases where we drop the assumption that $\ell$ is bounded below by a positive constant by invoking results from [14] (cf. Sect. 6.3).

Remark 2.4. Notice that the continuity of $v_{f, \ell, A}$ is part of the conclusion of Theorem 2.1. It will follow from the Ascoli-Arzelá theorem. For cases where $\ell$ does not depend on the input value, Theorem 2.1 remains true if we drop the requirement that $\|\beta\| \leq\|\alpha\|$ a.e. in Condition 3 of the colue definition.

Remark 2.5. In the paper [13], a more general version of Theorem 2.1 is announced (namely, Th. A) for cases where the controls for $f_{n}$ are allowed to take values in suitable compact sets $A_{n}$ containing $A$. However, the result of [13] requires $(f, \ell)$ to be coercively transient, it requires the nonexpansiveness condition, and it does not apply to cases where the Lagranigan $\ell(x, a)$ vanishes at some $x \in \mathbb{R}^{N}$ and $a \in A$. The proof of Theorem 2.1 below can easily be modified to cover cases where the control sets depend on $f_{n}$, in the following way. Assume there are compact spaces $A_{n}$ contained in a normed vector space with norm $\|\cdot\|$ such that $A_{n} \downarrow A$ in the Hausdorff distance sense ( $c f$. [2]) and such that $\ell \in C_{\mathrm{inc}}\left(\mathbb{R}^{N} \times A_{1}, \mathbb{R}_{+}\right)$, and replace Conditions $1-3$. in the colue definition with the following:

$1^{\prime} . f_{n} \in C_{\text {lip }}\left(\mathbb{R}^{N} \times A_{1}, \mathbb{R}^{N}\right)$ for all $n \in \mathbb{N}, \mathcal{R}:=\mathcal{R}_{f}(A) \equiv \mathcal{R}_{f_{n}}\left(A_{n}\right)$ is open, $f_{n} \rightarrow f$ uniformly on compact sets.

$2^{\prime} . \operatorname{Traj}\left(x, f_{n}, A_{n}, \mathcal{T}\right) \subseteq \operatorname{Traj}\left(x, f_{1}, A_{1}, \mathcal{T}\right)$ for each $n \in \mathbb{N}$ and $x \in \mathcal{R}$.

$3^{\prime}$. For each $p \in \mathbb{N}, x \in \mathcal{R}$, and $\phi$ in $\operatorname{Traj}_{\alpha}\left(x, f, \mathbb{R}^{N}\right)$, there is a measurable function $\beta:[0, \infty) \rightarrow A_{p}$ so that $\phi \in \operatorname{Traj}_{\beta}\left(x, f_{p}, \mathbb{R}^{N}\right)$. Moreover, if $\ell$ depends on the input value, then $\beta$ can be chosen so that $\|\alpha\| \geq\|\beta\|$ a.e.

Theorem 2.1 remains true if we change the definition of colues in this way and keep the other hypotheses of the theorem the same. The proof is similar to the proof we give in Section 4 below, except the approximating problems have the dynamics $f_{n}$ and the control set $A_{n}$, once we check that if $\alpha_{n}:[0, \infty) \rightarrow A_{n}$ is measurable for each $n$ and if $\alpha_{n} \rightarrow \bar{\alpha}$ weak- $\star$ (in the sense of relaxed controls valued on $A_{1}$ ), then $\bar{\alpha} \in \mathcal{A}$. (This is needed to verify that (4.9) below remains true under these more general hypotheses.) To check this, fix $t \geq 0$, and set

$$
\operatorname{dist}(a, A)=\inf \{\|a-p\|: p \in A\}
$$


for all $a \in A_{1}$. Since $A_{n} \downarrow A$, we get

$$
0 \leftarrow \int_{0}^{t} \int_{A_{n}} \operatorname{dist}(a, A) \mathrm{d}\left(\alpha_{n}(s)\right)(a) \mathrm{d} s=\int_{0}^{t} \int_{A_{1}} \operatorname{dist}(a, A) \mathrm{d}\left(\alpha_{n}(s)\right)(a) \mathrm{d} s \rightarrow \int_{0}^{t} \int_{A_{1}} \operatorname{dist}(a, A) \mathrm{d}(\bar{\alpha}(s))(a) \mathrm{d} s
$$

so $\operatorname{dist}(a, A)=0$ for almost all $a \in A_{1}$ and $s$ under the measure $\bar{\alpha}$, i.e., $\bar{\alpha}$ is supported on $A$. (One must also modify the proof that the Hamiltonians $F_{n}$ for the approximating problems $(c f .(4.1))$ converge to $H$ uniformly on compact sets, since the supremum in the definition of the $F_{n}$ 's is now over $A_{n}$. The proof of this convergence is in Section III.2 of [2]. The other changes needed in the proof of the theorem are slight.) In this way, the present paper includes the result stated in [13] and also extends this result to cases which are not covered by [13], including degenerate eikonal equations ( $c f$. Ex. 6.1 and Ex. 6.2 below) and Fuller's example ( $c f$. Sect. 6.3).

Remark 2.6. The requirements in Definition 2.2 and Definition 2.3 can be relaxed for problems which admit suitable optimal controls. More precisely, set

$$
O P T(p, S, h)=\bigcup_{\alpha \in \mathcal{A}}\left\{\phi \in \operatorname{Traj}_{\alpha}(p, h, S): v_{h, \ell, A}^{S}(p)=\int_{0}^{\tau_{S}(\phi)} \ell(\phi(s), \alpha(s)) \mathrm{d} s\right\}
$$

for all $h: \mathbb{R}^{N} \times A \rightarrow \mathbb{R}^{N}$ and $S \subseteq \mathbb{R}^{N}$. If we require $O P T(p, \mathcal{T}, f)$ to be nonempty for each $p \in \mathcal{R}$, then the theorem remains true if we change the definition of coercively transient so that only those trajectories

$$
\phi \in \operatorname{Traj}(x, f, A, \mathcal{T}) \cap O P T(x, T, f)
$$

are required to be coercifiable for each $x \in \mathcal{R}$. If we also assume $O P T\left(p,\{q\}, f_{n}\right) \neq \emptyset$ and $O P T(p,\{q\}, f) \neq \emptyset$ for all $p, q \in \mathcal{R}$ and $n \in \mathbb{N}$, then we can relax Condition 2 of Definition 2.2 to the requirement

$2^{\prime \prime} . \operatorname{Traj}\left(x, f_{n}, A, \mathcal{T}\right) \cap O P T\left(x, \mathcal{T}, f_{n}\right) \subseteq \operatorname{Traj}\left(x, f_{1}, A, \mathcal{T}\right)$ for each $n \in \mathbb{N}$ and $x \in \mathcal{R}$ and we can relax Condition 3 of the definition to

$3^{\prime \prime}$. For each $p \in \mathbb{N}$, each $q, x \in \mathcal{R}$, each $\alpha \in \mathcal{A}$, and each $\phi$ in $\operatorname{Traj}_{\alpha}(x, f,\{q\}) \cap O P T(p,\{q\}, f)$, there is an input $\beta:[0, \infty) \rightarrow A$ so that $\phi \in \operatorname{Traj}_{\beta}\left(x, f_{p},\{q\}\right)$ and so that $\|\alpha\| \geq\|\beta\|$ a.e.

The proof is similar to the proof we give in Section 4 below.

\section{Main Lemmas}

This section gives standard estimates from the theory of ordinary differential equations and lemmas on viscosity solutions and relaxed controls which we use to prove Theorem 2.1. We first recall the following basic ODE results on trajectories of Lipschitz dynamical laws (cf. Chap. 3 of [2]):

Lemma 3.1. Let $A$ be compact and $h \in C_{\text {lip }}\left(\mathbb{R}^{N} \times A, \mathbb{R}^{N}\right)$. Then for each $\alpha \in \mathcal{A}$, we have the following:

1. For each $x \in \mathbb{R}^{N}$, the system $\dot{x}=h(x, \alpha(t))$ has a unique solution trajectory starting at $x$ which is defined on $[0, \infty)$. This solution is denoted by $y_{x}^{h}(\cdot, \alpha)$.

2. For each $x \in \mathbb{R}^{N}$, there is an $M_{x}>0$ so that

$$
\left\|y_{x}^{h}(t, \alpha)-x\right\| \leq M_{x} t \text { for all } t \in\left[0,1 / M_{x}\right]
$$

e.g., $M_{x}:=\max \{\|h(z, a)\|:\|z-x\| \leq 1, a \in A\}+1$.

3. For each $x, z \in \mathbb{R}^{N}, \alpha \in \mathcal{A}$, and $t>0$,

$$
\left\|y_{x}^{h}(t, \alpha)-y_{z}^{h}(t, \alpha)\right\| \leq \mathrm{e}^{L t}\|x-z\| \text { and }\left\|y_{x}^{h}(t, \alpha)\right\| \leq(\|x\|+\sqrt{2 K t}) \mathrm{e}^{K t},
$$

where $L$ is the constant in the definition of Lipschitzness of $h$ and $K:=L+\sup \{\|h(0, a)\|: a \in A\}$. 
One easily checks that if $A$ is a compact metric space and if $h \in C_{\text {lip }}\left(\mathbb{R}^{N} \times A, \mathbb{R}^{N}\right)$, then the corresponding relaxed dynamics $h^{r}: \mathbb{R}^{N} \times A^{r} \rightarrow \mathbb{R}^{N}$ is also Lipschitz and continuous. In this case, it therefore makes sense to define $y_{x}^{h, r}(\cdot, \alpha)$ to be the unique solution of the dynamics $\dot{y}=h^{r}(y, \alpha)$ which starts at $x$ for each $\alpha \in \mathcal{A}^{r}$ ( $c f$. Sect. 2 for the notation). Notice for future reference that in that case, $y_{x}^{h, r}(\cdot, \alpha)$ is also a trajectory for the dynamics $h$ if we make the additional assumption that

$$
h(x, A):=\{h(x, a): a \in A\}
$$

is a convex set for all $x \in \mathcal{R}$ ( $c f$. the proof of Lem. 3.8 below).

The fact that $v_{f, \ell, A}$ is the minimal viscosity solution of $(2.3)$ that satisfies the side condition $(S C)$ when the hypotheses of Theorem 2.1 are satisfied will be a consequence of the following completeness characterization for HJBE's for exit time problems with Lipschitz dynamics:

Lemma 3.2. Assume the following:

1. $A$ is a compact topological space, and $\mathcal{T} \subseteq \mathbb{R}^{N}$ is closed.

2. $h \in C_{\text {lip }}\left(\mathbb{R}^{N} \times A, \mathbb{R}^{N}\right)$ and $T_{A, h}$ is continuous.

3. $\ell \in C\left(\mathbb{R}^{N} \times A, \mathbb{R}\right)$ is bounded below by a positive constant $m$.

4. $\mathcal{R}_{h}$ is open.

If $v_{h, \ell, A}$ is continuous on $\mathcal{R}_{h}$, then it is the complete viscosity solution of the corresponding HJBE

$$
\sup _{a \in A}\{-h(x, a) \cdot D v(x)-\ell(x, a)\}=0
$$

on $\mathcal{R}_{h} \backslash \mathcal{T}$ in the class of functions $w \in C\left(\mathcal{R}_{h}\right)$ which vanish on $\mathcal{T}$, which are bounded below, and which satisfy $\lim _{x \rightarrow x_{o}} w(x)=+\infty$ for each $x_{o} \in \partial\left(\mathcal{R}_{h}\right)$.

Proof. A proof for the case where $\ell$ is bounded above and $\mathcal{T}$ has compact boundary is given in [2]. The analog for cases where $\ell$ is not bounded above and $\partial \mathcal{T}$ is not compact is proven similarly (cf. [15]), save for the part where one must show that for each $x_{o} \in \partial\left(\mathcal{R}_{h}\right)$ we have $v_{h, \ell, A}(x) \rightarrow+\infty$ as $\mathcal{R}_{h} \ni x \rightarrow x_{o}$. The proof of that part is an elementary localization argument which is similar to arguments from [2]. Indeed, fix $x_{o} \in \partial\left(\mathcal{R}_{h}\right)$, and set

$$
T(p)=\inf \{\tau(\phi): \phi \in \operatorname{Traj}(p, h, A, \mathcal{T})\}
$$

for each $p \in \mathcal{R}_{h}$. Suppose for the sake of obtaining a contradiction that there is a constant $M<\infty$ and points $x_{n} \in \mathcal{R}_{h}$ for which $\left\|x_{n}-x_{0}\right\|<1 / n$ and $T\left(x_{n}\right) \leq M$ for all $n$. Let $L$ be as in the definition of Lipschitzness of $h$, and pick $R>0$ so that $y_{p}^{h}(t, \beta) \in B_{R}\left(x_{o}\right)$ for all $t \in[0, M+1]$, all $p \in B_{1}\left(x_{o}\right)$, and all $\beta \in \mathcal{A}$. Such an $R$ exists by the estimates of Lemma 3.1. Fix $\delta<R / 2$, and choose $\bar{n} \in \mathbb{N}$ so that $\mathrm{e}^{L(M+1)} / \bar{n}<\delta$. Use the infimum definition to find a $\bar{\alpha} \in \mathcal{A}$ so that

$$
\bar{t}:=\tau\left(y_{x_{\bar{n}}}^{h}(\cdot, \bar{\alpha})\right)<T\left(x_{\bar{n}}\right)+1 .
$$

The third conclusion of the previous lemma gives

$$
\left\|y_{x_{o}}^{h}(\bar{t}, \bar{\alpha})-y_{x_{\bar{n}}}^{h}(\bar{t}, \bar{\alpha})\right\| \leq\left\|x_{o}-x_{\bar{n}}\right\| \mathrm{e}^{L \bar{t}}<\delta,
$$

so $\operatorname{dist}\left(y_{x_{o}}^{h}(\bar{t}, \bar{\alpha}), \mathcal{T}_{R}\right)<\delta$, where

$$
T_{R}:=\mathcal{T} \cap \overline{B_{R}\left(x_{o}\right)} .{ }^{2}
$$

\footnotetext{
${ }^{2}$ Recall that for $U, V \subset \mathbb{R}^{N}$, we set $\operatorname{dist}(U, V)=\inf \{\|u-b\|: u \in U, b \in V\}$. We write $\operatorname{dist}(u, V)$ when $U=\{u\}$ instead of $\operatorname{dist}(\{u\}, V)$.
} 
Since $\mathcal{T}_{R} \subset \mathcal{R}_{h}$ is compact and $\mathcal{R}_{h}$ is open, there is an $\varepsilon>0$ so that $\mathcal{T}_{R}^{\varepsilon} \subseteq \mathcal{R}_{h} .{ }^{3}$. Since we could have chosen $\delta<\varepsilon$ as well (by enlarging $\bar{n}$ ), it follows that $y_{x_{o}}^{h}(\bar{t}, \bar{\alpha}) \in \mathcal{R}_{h}$, so $x_{o} \in \mathcal{R}_{h}$, which contradicts the fact that $\mathcal{R}_{h}$ is open. Therefore, $T(p) \rightarrow+\infty$ as $\mathcal{R}_{h} \ni x \rightarrow x_{o}$. The limit condition now follows since $v_{h, \ell, A}(p) \geq m T(p)$ for all $p \in \mathcal{R}_{h}$.

We remark for future reference that if $v_{h, \ell, A}$ is continuous on $\mathcal{R}_{h}$, then it is still a viscosity solution of (3.1) on $\mathcal{R}_{h}(A) \backslash \mathcal{T}$ if we allow $m=0$ in the hypotheses of the previous lemma (cf. [2]). However, as we saw in the introduction, the completeness characterization can fail if $m=0$.

We also need the following "one-sided" local analogue of the previous lemma which is a special case of Theorem IV.4.1 of [2]:

Lemma 3.3. Assume the following conditions are satisfied:

1. $A$ is a compact space and $\omega_{o} \in \mathbb{R} \cup\{+\infty\}$.

2. $\mathcal{T} \subset \mathbb{R}^{N}$ is closed and $\Omega \subset \mathbb{R}^{N}$ is open.

3. $h \in C_{\text {lip }}\left(\mathbb{R}^{N} \times A, \mathbb{R}^{N}\right)$, and $\ell \in C\left(\mathbb{R}^{N} \times A, \mathbb{R}\right)$ is bounded below by a positive constant.

4. $w \in C(\Omega)$ is a viscosity supersolution of (3.1) on $\Omega \backslash \mathcal{T}$ which is bounded below.

5. $w \geq 0$ on $\Omega \cap \mathcal{T}$, and $w<\omega_{o}$ on $\Omega$.

6. $\lim _{\Omega \ni x \rightarrow x_{o}} w(x)=\omega_{o}$ for all $x_{o} \in \partial \Omega$.

Then $w \geq v_{h, \ell, A}$ on $\Omega$.

We will use the following stability lemma ( $c f .[2])$ :

Lemma 3.4. Let $F_{n}, F \in C\left(\mathbb{R}^{N} \times \mathbb{R}^{N}, \mathbb{R}\right)$ for all $n \in \mathbb{N}$, let $\Omega \subseteq \mathbb{R}^{N}$ be open, and assume that $u_{n}: \Omega \rightarrow \mathbb{R}$ is a viscosity solution of $F_{n}\left(x, D u_{n}(x)\right)=0$ on $\Omega$ for each $n$. Assume the following conditions hold:

1. $u_{n} \rightarrow u$ locally uniformly on $\Omega$.

2. $F_{n} \rightarrow F$ locally uniformly on $\Omega \times \mathbb{R}^{N}$.

Then $u$ is a viscosity solution of $F(x, D u(x))=0$ on $\Omega$.

To show that $v_{f, \ell, A}$ is the maximal viscosity solution of the HJBE (2.3) that satisfies the side condition (SC) when the hypotheses of Theorem 2.1 hold, we use the following partial converse of the dynamic programming principle ( $c f .[2])$ :

Lemma 3.5. Let $A$ be a compact topological space, let $h \in C\left(\mathbb{R}^{N} \times A, \mathbb{R}^{N}\right)$, let $\ell \in C\left(\mathbb{R}^{N} \times A\right.$, $\left.\mathbb{R}\right)$, and let $E \subseteq \mathbb{R}^{N}$ be open and bounded. Assume that $u \in C(\bar{E})$ is a viscosity subsolution of the equation

$$
\sup _{a \in A}\{-h(x, a) \cdot D u(x)-\ell(x, a)\}=0
$$

on $E$ and that $h$ is Lipschitz on $E$. Set $\tau_{q}(\beta)=\inf \left\{t \geq 0: y_{q}^{h}(t, \beta) \in \partial E\right\}$ for each $\beta \in \mathcal{A}$ and $q \in E$. Then

$$
u(q) \leq \int_{0}^{r} \ell\left(y_{q}^{h}(s, \beta), \beta(s)\right) \mathrm{d} s+u\left(y_{q}^{h}(r, \beta)\right)
$$

for $0 \leq r<\tau_{q}(\beta)$, all $\beta \in \mathcal{A}$, and all $q \in E$.

\footnotetext{
${ }^{3}$ Recall that for any $S \subseteq \mathbb{R}^{N}$ and any $\varepsilon>0, S^{\varepsilon}=\left\{p \in \mathbb{R}^{N}: \inf _{x \in S}\|x-p\|<\varepsilon\right\}$. We write $B_{\varepsilon}(p)$ when $S=\{p\}$ instead of $\{p\}^{\varepsilon}$.
} 
We also use the following analogue for viscosity supersolutions of (2.3) from [2]:

Lemma 3.6. Let $A, h, \ell$, and $E$ satisfy the assumptions of Lemma 3.5, and let $w \in C(\bar{E})$ be a viscosity supersolution of $(3.2)$ on $E$. Set

$$
T_{\delta}(p):=\inf _{\alpha \in \mathcal{A}}\left\{t: \operatorname{dist}\left(y_{p}^{h}(t, \alpha), \partial E\right) \leq \delta\right\}
$$

for each $p \in E$ and $\delta>0$. Then

$$
w(p) \geq \inf _{\alpha \in \mathcal{A}}\left\{\int_{0}^{t} \ell\left(y_{p}^{h}(s, \alpha), \alpha(s)\right) \mathrm{d} s+w\left(y_{p}^{h}(t, \alpha)\right)\right\}
$$

for all $t \in\left(0, T_{\delta}(p)\right), p \in E$, and $\delta \in(0, \operatorname{dist}(p, \partial E) / 2]$.

Notice for future reference that the previous two lemmas do not require $\ell$ to be bounded below by a positive constant. We will use the following classical compactness lemma from [24]:

Lemma 3.7. Let $A$ be a compact metric space, and let $\left\{\alpha_{n}\right\}_{n=1}^{\infty}$ in $\mathcal{A}^{r}$ and $c>0$ be given. Assume $h$ : $\mathbb{R}^{N} \times A \rightarrow \mathbb{R}^{N}$ is a Lipschitz dynamical law. Then there is a subsequence of $\left\{\alpha_{n}\right\}_{n=1}^{\infty}$ (which we do not relabel) and an $\alpha \in \mathcal{A}^{r}$ such that the following conditions hold:

1. $\alpha_{n} \rightarrow \alpha$ weak-star on $[0, c]$.

2. If $x_{n} \rightarrow x$ in $\mathbb{R}^{N}$, then $y_{x_{n}}^{h, r}\left(\cdot, \alpha_{n}\right) \rightarrow y_{x}^{h, r}(\cdot, \alpha)$ uniformly on $[0, c]$.

Lemma 3.7 follows from the sequential compactness of $\mathcal{A}^{r}$ and the Bellman-Gronwall Inequality (cf. [24]). We also need the following characterization of relaxed trajectories. The proof is a variant of the proof of Corollary VI.1.4 of [2] which is based on the Filippov Selection theorem. We use it in conjunction with the previous lemma by first extracting weak- $\star$ limits of sequences in $\mathcal{A}$ and then arguing that the trajectories for $f^{r}$ and the weak- $\star$ limits are also trajectories for $f$.

Lemma 3.8. Let $A$ be a compact metric space and let $h: \mathbb{R}^{N} \times A \rightarrow \mathbb{R}^{N}$ and $\ell: \mathbb{R}^{N} \times A \rightarrow \mathbb{R}$ be continuous functions such that $h(x, A) \times \ell(x, A)$ is convex for all $x \in \mathbb{R}^{N 4}$. Let $\left(\phi^{r}, \mu^{r}\right)$ be a trajectory-input pair for $h^{r}$. Then there is a measurable mapping $\alpha:[0, \infty) \rightarrow A$ so that

$$
\int_{0}^{t} \ell^{r}\left(\phi^{r}(s), \mu^{r}(s)\right) \mathrm{d} s=\int_{0}^{t} \ell\left(\phi^{r}(s), \alpha(s)\right) \mathrm{d} s \text { for all } t \geq 0
$$

and so that $\left(\phi^{r}, \alpha\right)$ is a trajectory-input pair for $h$.

Proof. It suffices to show that $h^{r}\left(x, A^{r}\right) \times \ell^{r}\left(x, A^{r}\right)=h(x, A) \times \ell(x, A)$ for all $x$. Indeed, if that is the case, then for each $s \geq 0$, there is an $\alpha(s) \in A$ so that

$$
h\left(\phi^{r}(s), \alpha(s)\right)=\int_{A} h\left(\phi^{r}(s), a\right) \mathrm{d} \mu_{s}^{r}(a) \quad \text { and } \quad \ell\left(\phi^{r}(s), \alpha(s)\right)=\int_{A} \ell\left(\phi^{r}(s), a\right) \mathrm{d} \mu_{s}^{r}(a) .
$$

Applying the Filippov-Castaing Theorem ( $c f$. [6] or Th. I.7.10 of [24]), we can assume $s \mapsto \alpha(s)$ is a measurable function, and then the result follows once we integrate both sides of the equations in (3.5). The inclusion " $\supseteq$ " follows since $A^{r}$ includes point-mass Radon probability measures. By the Mean Value Theorem (cf. [2], Sect. III.2), if $m \in A^{r}$ and $G: A \rightarrow \mathbb{R}^{N+1}$ is $m$-measurable, then $\int_{A} G(a) \mathrm{d} m(a) \in \overline{c o} G(A)$, and this gives the reverse inclusion, by the convexness assumption and the continuity of the mappings $a \mapsto(h(x, a), \ell(x, a))$.

\footnotetext{
${ }^{4}$ We let $h(x, A) \times \ell(x, A)$ denote $\{(h(x, a), \ell(x, a)): a \in A\}$ and $h^{r}\left(x, A^{r}\right) \times \ell^{r}\left(x, A^{r}\right):=\left\{\left(h^{r}(x, a), \ell^{r}(x, a)\right): a \in A^{r}\right\}$. Also, we sometimes write $\alpha_{s}$ instead of $\alpha(s)$ when $s \mapsto \alpha(s) \in A^{r}$.
} 


\section{Proof of Theorem 1}

Let $f_{n}$ be as in the colue definition. We set $v:=v_{f, \ell, A}, v_{n}:=v_{f_{n}, \ell, A}, v^{p}=v_{f, \ell, A}^{p}$, and $v_{n}^{p}=v_{f_{n}, \ell, A}^{p}$ for all $p \in \mathcal{R}$ and $n \in \mathbb{N}$ in the sequel.

By Condition 1 of Definition 2.2 and Lemma 3.2, $v_{n}$ is the complete viscosity solution of

$$
\sup _{a \in A}\left\{-f_{n}(x, a) \cdot D u(x)-\ell(x, a)\right\}=0
$$

on $\mathcal{R} \backslash \mathcal{T}$ in the class of functions satisfying the side condition (SC) if it is continuous on $\mathcal{R}$. We will presently show that the $v_{n}$ 's are continuous on $\mathcal{R}$. Since $F_{n}(x, p):=\sup \left\{-f_{n}(x, a) \cdot p-\ell(x, a): a \in A\right\}$ is continuous for each $n$ and $f_{n} \rightarrow f$ locally uniformly, we will then be able to conclude from Lemma 3.4 that $v$ is a viscosity solution of the HJBE (2.3) on $\mathcal{R} \backslash \mathcal{T}$ if $v_{n} \rightarrow v$ uniformly on compact subsets of $\mathcal{R}$. We now prove the continuity of the $v_{n}$ 's and the locally uniform convergence $v_{n} \rightarrow v$ using the Ascoli-Arzelá theorem (cf. [10]). The argument is similar in spirit to the proof of Corollary III.2.22 of [2], but extra care is needed because $f$ is allowed to be non-Lipschitz.

We first establish the pointwise boundedness of the $v_{n}$ 's. Notice that for each $p \in \mathcal{R}$ and each $r \in \mathbb{N}$, we have $v_{r}^{p} \leq v^{p}$ pointwise. Indeed, if $x, p \in \mathcal{R}, r \in \mathbb{N}$, and $\phi \in \operatorname{Traj}_{\alpha}(x, f,\{p\})$, then we can use the definition of colues to find an input $\beta \in \mathcal{A}$ (depending on $r$ ), with $\|\beta\| \leq\|\alpha\|$ a.e., so that $\phi \in \operatorname{Traj}_{\beta}\left(x, f_{r},\{p\}\right.$ ). Since $\ell \in C_{\text {inc }}\left(\mathbb{R}^{N} \times A, \mathbb{R}\right)$ for all $r$, we get

$$
\int_{0}^{\tau_{p}(\phi)} \ell(\phi(s), \alpha(s)) \mathrm{d} s \geq \int_{0}^{\tau_{p}(\phi)} \ell(\phi(s), \beta(s)) \mathrm{d} s \geq v_{r}^{p}(x),
$$

so the claim follows by infimizing over $\phi \in \operatorname{Traj}_{\alpha}(x, f,\{p\})$ and all $\alpha \in \mathcal{A}$ on the left side of (4.2). Therefore,

$$
v_{n}(x)=\inf _{p \in \mathcal{T}} v_{n}^{p}(x) \leq \inf _{p \in \mathcal{T}} v^{p}(x)=v(x)
$$

for all $x \in \mathcal{R}$ and $n \in \mathbb{N}$, as desired.

Next we check the equicontinuity of the $v_{n}$ 's. Fix $x \in \mathcal{R} \backslash \mathcal{T}$, and let $\delta>0$ be such that $B_{\delta}(x) \subset \mathcal{R} \backslash \mathcal{T}$. By the continuity of $T_{A, f}$, we can travel between any points $y, z \in B_{\delta}(x)$ using the dynamics $f$. This and Condition 3 from Definition 2.2 gives $v_{n}(z)-v_{n}(y) \leq v_{n}^{y}(z) \leq v^{y}(z)$ for all $y, z \in B_{\delta}(x)$ and all $n \in \mathbb{N}$. (We use the definition of the infimum and the fact that $y, z \in \mathcal{R}$ to get the first inequality.) Arguing symmetrically, we get

$$
\left|v_{n}(z)-v_{n}(y)\right| \leq v^{y}(z) \vee v^{z}(y)
$$

for all $y, z \in B_{\delta}(x)$ and all $n \in \mathbb{N}$.

Notice also that $(p, z) \mapsto v^{z}(p)$ is continuous on $\mathcal{R} \times \mathcal{R}$. Indeed, let $\varepsilon, \hat{\delta}>0$ and $x, y \in \mathcal{R}$ be given. Since $T_{A, f}$ is continuous, there is a $\mu=\mu(\hat{\delta})>0$ so that if

$$
\tilde{x}, \tilde{y} \in \mathcal{R} \text { and }\|x-\tilde{x}\| \vee\|y-\tilde{y}\|<\mu(\hat{\delta})
$$

then there are inputs $\alpha_{1}$ and $\alpha_{2}$ in $\mathcal{A}$, numbers $t_{1}, t_{2} \in[0, \hat{\delta})$, and trajectories $\phi_{1} \in \operatorname{Traj}_{\alpha_{1}}(x, f,\{\tilde{x}\})$ and $\phi_{2} \in$ $\operatorname{Traj}_{\alpha_{2}}(\tilde{y}, f,\{y\})$ such that $\phi_{1}\left(t_{1}\right)=\tilde{x}$ and $\phi_{2}\left(t_{2}\right)=y$. Using the definition of the infimum and concatenating trajectories and controls, we get

$$
v^{y}(x)-v^{\tilde{y}}(\tilde{x})-\varepsilon / 2 \leq \int_{0}^{t_{1}} \ell\left(\phi_{1}(s), \alpha_{1}(s)\right) \mathrm{d} s+\int_{0}^{t_{2}} \ell\left(\phi_{2}(s), \alpha_{2}(s)\right) \mathrm{d} s .
$$

Since $(f, \ell)$ is a Lipschitz upper envelope, we know that $\phi_{j}$ is a trajectory for $f_{1}$ for $j=1,2$, where $f_{1}$ is Lipschitz. Using the estimates of Lemma 3.1, we conclude that $\|\gamma(s)\|$ is uniformly bounded over the restriction to [0,1] 
of all trajectories $\gamma$ of $f$ starting at points $p$ with $\|p-x\| \wedge\|p-y\| \leq 1$. Let $\kappa$ be any such uniform bound, set $\hat{B}:=\sup \ell\left\lceil\left[\overline{B_{\kappa}(0)} \times A\right]\right.$, and pick $\hat{\delta}>0$ such that $\hat{\delta}<\frac{\varepsilon}{4[\hat{B}+1]} \wedge 1$ and a corresponding $\mu=\mu(\hat{\delta})<1$. Then (4.5) and (4.6) give

$$
v^{y}(x)-v^{\tilde{y}}(\tilde{x})-\varepsilon / 2 \leq \frac{\varepsilon}{4(\hat{B}+1)}(\hat{B}+\hat{B})
$$

so $v^{y}(x)-v^{y}(\tilde{x}) \leq \varepsilon$. By symmetry, we get

$$
\|x-\tilde{x}\| \vee\|y-\tilde{y}\|<\delta_{\varepsilon} \Rightarrow\left|v^{y}(x)-v^{\tilde{y}}(\tilde{x})\right| \leq \varepsilon
$$

for some $\delta_{\varepsilon}>0$, so $(p, q) \mapsto v^{q}(p)$ is continuous on $\mathcal{R} \times \mathcal{R}$, as claimed.

It now follows from $(4.3,4.4)$, and $(4.7)$ that the $v_{n}$ 's are locally equicontinuous and pointwise bounded on $\mathcal{R}$. In particular, the $v_{n}$ 's are viscosity solutions of the corresponding HJBE's (4.1) on $\mathcal{R} \backslash \mathcal{T}$. Therefore, we can apply the Ascoli-Arzelà theorem (on $\overline{B_{\delta / 2}(x)}$, for example, where $\delta$ is chosen so that $B_{\delta / 2}(x) \subseteq \mathcal{R} \backslash \mathcal{T}$ ) to get a locally defined function $\bar{v}$ such that $v_{n} \rightarrow \bar{v}$ on $\overline{B_{\delta / 2}(x)}$ uniformly, at least along a subsequence (cf. [10]). These locally defined functions $\bar{v}$ are (local) solutions of the HJBE, by stability ( $c f$. Lem. 3.4).

Fixing $x \in \mathcal{R} \backslash \mathcal{T}$ and $\delta$ as above and $p \in B_{\delta / 2}(x) \subseteq \mathcal{R} \backslash \mathcal{T}$ and letting $\bar{v}$ denote the locally defined function on $\overline{B_{\delta / 2}(x)}$, we now show that $\bar{v}=v$ (which will show that $v$ is a viscosity solution of the HJBE on $B_{\delta / 2}(x)$, hence on all of $\mathcal{R} \backslash \mathcal{T})$. Since $v_{n}(p) \leq v(p)$ for all $n, \bar{v}(p) \leq v(p)$. We assume that $v_{n}(p) \rightarrow \bar{v}(p)$, possibly by passing to a subsequence without relabelling. To prove the reverse inequality, let $\varepsilon>0$ be given, and choose inputs $\alpha_{n} \in \mathcal{A}$ and trajectories $\phi_{n} \in \operatorname{Traj}_{\alpha_{n}}\left(p, f_{n}, \mathcal{T}\right)$ for each $n \in \mathbb{N}$ so that

$$
v_{n}(p)+\varepsilon \geq \int_{0}^{\tau\left(\phi_{n}\right)} \ell\left(\phi_{n}(s), \alpha_{n}(s)\right) \mathrm{d} s
$$

for all $n$. This is possible since $\mathcal{R}_{f_{n}}=\mathcal{R}_{f}$ for all $n$. Since $v_{n}(p) \leq v(p)$ for all $n$ and $\ell$ is bounded below by a positive constant, it follows that the exit times $\tau\left(\phi_{n}\right)$ of the $\phi_{n}$ are bounded above, so we can assume (by passing to a subsequence, if necessary, without relabeling) that $\tau\left(\phi_{n}\right) \rightarrow \mu \in \mathbb{R}$. We can also find inputs $\beta_{n} \in \mathcal{A}$ so that $\left(\phi_{n}, \beta_{n}\right)$ is a trajectory-control pair for the dynamics $f_{1}$, by Condition 2 of the colue definition. Also note that, by the estimates in Lemma 3.1 applied to the dynamics $f_{1},\left\|\phi_{n}(s)\right\|$ is bounded as $s$ varies over $[0, \mu+1]$ and $n$ varies in $\mathbb{N}$ (since the $\phi_{n}$ 's are trajectories of the Lipschitz dynamics $f_{1}$ ). Applying Lemma 3.7, we know that there is a weak- ${ }^{\star}$ limit of a subsequence of the $\beta_{n}$ 's, which we call $\bar{\beta}$, so that the $\phi_{n}$ 's converge uniformly on $[0, \mu+1]$ to a relaxed trajectory for $f_{1}^{r}(x, \bar{\beta})$. Let $\phi^{r}$ denote this relaxed trajectory. Passing to a further subsequence if necessary (without relabeling), we can also assume that $\alpha_{n} \rightarrow \bar{\alpha} \in \mathcal{A}$ weak-*.

We conclude that

$$
\phi^{r}(t) \leftarrow \phi_{n}(t)=p+\int_{0}^{t} f_{n}\left(\phi_{n}(s), \alpha_{n}(s)\right) \mathrm{d} s \rightarrow p+\int_{0}^{t} f^{r}\left(\phi^{r}(s), \bar{\alpha}(s)\right) \mathrm{d} s
$$

for any $t \geq 0$ and that $\left(\phi^{r}, \bar{\alpha}\right)$ is a trajectory-input pair for $f^{r}$. (Here and below, the right arrow follows from an application of the Dominated Convergence theorem. The elementary arguments will be omitted. For similar arguments, see the proof of Claim 7.3 in the Appendix.) Now use Lemma 3.8 to find an $\alpha \in \mathcal{A}$ so that $\left(\phi^{r}, \alpha\right)$ is a trajectory-input pair for $f$ which gives the same running costs as $\left(\phi^{r}, \bar{\alpha}\right)$. This gives

$$
v_{n}(p)+\varepsilon \geq \int_{0}^{\tau\left(\phi_{n}\right)} \ell\left(\phi_{n}(s), \alpha_{n}(s)\right) \mathrm{d} s \rightarrow \int_{0}^{\mu} \ell^{r}\left(\phi^{r}(s), \bar{\alpha}(s)\right) \mathrm{d} s=\int_{0}^{\mu} \ell\left(\phi^{r}(s), \alpha(s)\right) \mathrm{d} s \geq v(p),
$$

since $\mathcal{T} \ni \phi_{n}\left(\tau\left(\phi_{n}\right)\right) \rightarrow \phi^{r}(\mu)$ and $\mathcal{T}$ is closed. Recall that $v(p) \geq v_{m}(p)$ for all $m \in \mathbb{N}$ (by (4.3)). Therefore, for each $p \in \overline{B_{\delta / 2}(x)}$, we have $v_{n}(p) \rightarrow v(p)$ and $v_{n}(p) \rightarrow \bar{v}(p)$ along a subsequence, so $\bar{v}=v$ on $\overline{B_{\delta / 2}(x)}$. Since 
$\bar{v}$ is a viscosity solution of $(2.3)$ on $B_{\delta / 2}(x)$, it follows that $v$ is a viscosity solution of (2.3) on $\mathcal{R} \backslash \mathcal{T}$ (since the definition of viscosity solutions is a local one and $x \in \mathcal{R} \backslash \mathcal{T}$ was arbitrary).

We turn next to the uniqueness characterization. Let $w \in C(\mathcal{R})$ be another viscosity solution of the HJBE on $\mathcal{R} \backslash \mathcal{T}$ satisfying the condition $(S C)$. Then, $w$ is also a viscosity supersolution of (4.1) on $\mathcal{R} \backslash \mathcal{T}$ for $n$ large enough. Indeed, let $x \in \mathcal{R} \backslash \mathcal{T}$ be such that $D^{-} w(x) \neq \emptyset$, and pick $p \in D^{-} w(x)$. Let $\bar{a}$ be as in Condition 4 of Definition 2.2. This gives

$$
0 \leq-f(x, \bar{a}) \cdot p-\ell(x, \bar{a}) \leq-f_{n}(x, \bar{a}) \cdot p-\ell(x, \bar{a}) \leq \sup _{a \in A}\left\{-f_{n}(x, a) \cdot p-\ell(x, a)\right\}
$$

for all $n \in \mathbb{N}$. It follows that $w$ is a viscosity supersolution of (4.1) on $\mathcal{R} \backslash \mathcal{T}$. By $(4.3), v$ satisfies the limit condition in (SC). By completeness ( $c f$. Lem. 3.2), we get $w \geq v_{n}$ pointwise, so $w \geq v$ pointwise, since $v_{n} \rightarrow v$ pointwise along a subsequence.

To prove the reverse inequality, let $x \in \mathcal{R} \backslash \mathcal{T}, \alpha \in \mathcal{A}$, and $\phi \in \operatorname{Traj}_{\alpha}(x, f, \mathcal{T})$ be given. Set

$$
\bar{t}:=\sup \left\{t \in[0, \tau(\phi)]: w(x) \leq \int_{0}^{t} \ell(\phi(s), \alpha(s)) \mathrm{d} s+w(\phi(t))\right\}
$$

and let us suppose that $\bar{t}<\tau(\phi)$, for the sake of obtaining a contradiction. Notice that $\phi(\bar{t}) \in \mathcal{R} \backslash \mathcal{T}$. Since $\phi$ and $\ell$ are continuous and $w \in C(\mathcal{R})$, we know that

$$
w(x) \leq \int_{0}^{\bar{t}} \ell(\phi(s), \alpha(s)) \mathrm{d} s+w(\phi(\bar{t}))
$$

Since $(f, \ell)$ is coercively transient, we also know that $\phi(\cdot+\bar{t})$ is a coercifiable trajectory at $\phi(\bar{t})$. Therefore, we can find $t, t^{\prime} \in(0, \tau(\phi(\cdot+\bar{t})))$, an input $\beta \in \mathcal{A}$, and a trajectory $\psi \in \operatorname{Traj}_{\beta}(\phi(\bar{t}), f, \mathcal{T})$ so that $\psi\left(t^{\prime}\right)=\phi(t+\bar{t})$ and

$$
\int_{0}^{t^{\prime}} \ell(\psi(s), \beta(s)) \mathrm{d} s \leq \int_{0}^{t} \ell(\phi(s+\bar{t}), \alpha(s+\bar{t})) \mathrm{d} s=\int_{\bar{t}}^{t+\bar{t}} \ell(\phi(s), \alpha(s)) \mathrm{d} s,
$$

and a number $N \in \mathbb{N}$ and open sets $\left\{S_{n}\right\}_{n=N}^{\infty}$ so that for all $n \geq N$,

$$
f\left\lceil[ S _ { n } \times A ] \in C _ { \text { lip } } ( S _ { n } \times A ) \text { and } S _ { n } \supset \text { Trace } \psi \left\lceil\left[1 / n, t^{\prime}\right] .\right.\right.
$$

Replacing the $S_{n}$ 's by smaller bounded sets as needed and applying Lemma 3.5, we get

$$
w(\psi(1 / n)) \leq \int_{1 / n}^{t^{\prime}} \ell(\psi(s), \beta(s)) \mathrm{d} s+w(\phi(t+\bar{t})) .^{5}
$$

Letting $n \rightarrow \infty$ in (4.12) and using the facts that $w \in C(\mathcal{R} \backslash \mathcal{T})$ and $\psi(0)=\phi(\bar{t})$, we conclude that

$$
w(\phi(\bar{t})) \leq \int_{0}^{t^{\prime}} \ell(\psi(s), \beta(s)) \mathrm{d} s+w(\phi(t+\bar{t}))
$$

\footnotetext{
${ }^{5}$ Since $\phi(t+\bar{t})=\psi\left(t^{\prime}\right) \in \mathcal{R}, \psi$ stays in $\mathcal{R}$ on $\left[0, t^{\prime}\right]$. It could be that $\psi$ reaches $\mathcal{T}$ on $\left[0, t^{\prime}\right]$. However, since $w \equiv 0$ on $\mathcal{T}$ and $\ell \geq 0$, we can assume that $\psi\left\lceil\left[1 / n, t^{\prime}\right]\right.$ stays in $\mathcal{R} \backslash \mathcal{T}$, so $w$ is a viscosity solution of $(2.3)$ on the closure of an open set containing $\psi\left\lceil\left[1 / n, t^{\prime}\right]\right.$. Indeed, if $\psi$ reaches $\mathcal{T}$ on $\left[0, t^{\prime}\right]$, and if $\bar{s} \in\left(0, t^{\prime}\right)$ is the supremum of the times $p \in\left[0, t^{\prime}\right]$ so that $\psi(p) \in \mathcal{T}$, then $0 \leq \bar{s}_{\bar{s}} \ell(\psi(s), \beta(s)) \mathrm{d} s+w(\phi(t+\bar{t}))$, while if $\tilde{s}$ is the infimum of those times, then $w(\psi(1 / n)) \leq{ }_{1 / n}^{\tilde{s}} \ell(\psi(s), \beta(s)) \mathrm{d} s$. Now add these inequalities, then add in the extra running costs, which are nonnegative since $\ell$ is nonnegative, to get (4.12) for cases where $\psi$ reaches $\mathcal{T}$ on $\left[0, t^{\prime}\right]$ as well.
} 
Combining $(4.10,4.11)$, and (4.13), we get

$w(x) \leq \int_{0}^{\bar{t}} \ell(\phi(s), \alpha(s)) \mathrm{d} s+\int_{0}^{t^{\prime}} \ell(\psi(s), \beta(s)) \mathrm{d} s+w(\phi(t+\bar{t})) \leq \int_{0}^{t+\bar{t}} \ell(\phi(s), \alpha(s)) \mathrm{d} s+w(\phi(t+\bar{t}))$.

Since $\bar{t}<t+\bar{t}<\tau(\phi)$, this contradicts the definition of $\bar{t}$. Therefore, $\bar{t}=\tau(\phi)$. The inequality " $w \leq v$ " now follows by infimizing over all $\alpha$ 's that drive $x$ to $\mathcal{T}$ using $f$ and the corresponding $\phi$ 's in $(4.10)$, since $w \equiv 0$ on $\mathcal{T}$. We conclude that $v$ is the unique viscosity solution of the corresponding HJBE on $\mathcal{R} \backslash \mathcal{T}$ in the class of functions $w \in C(\mathcal{R})$ satisfying the condition $(S C)$, as desired.

\section{Reflected Brachystochrone Problem}

Before turning to variants of Theorem 2.1 which apply under weaker hypotheses, we show how Theorem 2.1 gives a uniqueness characterization for Sussmann's Reflected Brachystochrone Problem (RBP). Sussmann's RBP is a variant of Bernoulli's Brachystochrone Problem (BP). The BP is as follows: for each $\Lambda \in \mathbb{R}$ and each pair of points of $p_{o}$ and $p_{f}$ in the half plane $\left\{(x, y)^{\prime}: y \geq-\Lambda\right\}$, find a Lipschitz function $f=\left(f_{1}, f_{2}\right):[0, T] \rightarrow \mathbb{R}^{2}$ which satisfies $f(0)=p_{o}, f(T)=p_{f}$, and

$$
\frac{1}{2}\left[\left|\dot{f}_{1}(t)\right|^{2}+\left|\dot{f}_{2}(t)\right|^{2}\right]=g\left[\Lambda+f_{2}(t)\right] \text { a.e. } t
$$

and is such that $T$ has the least possible value. The dynamics is the motion of a particle traveling between two points in a vertical plane and $g$ is the gravitational constant. The solution trajectory was derived by Bernoulli in 1697 for each choice of $p_{o}$ and $p_{f}$. When $\Lambda=0$, it is the curve traced out by a point $p$ of a circle that rolls without slipping along the $x$-axis in such a way that $p$ passes from $p_{o}$ to $p_{f}$ without touching the $x$-axis in between. These trajectories are cycloids and therefore have the form

$$
x(\phi)=x_{o}+\frac{D}{2}(\phi-\sin \phi), \quad y(\phi)=\frac{D}{2}(1-\cos \phi), \quad 0 \leq \phi \leq 2 \pi .
$$

Bernoulli proved his result using Fermat's Minimal Time Principle, Snell's light refraction law, and a discretization technique ( $c f$. [21]). If we permit $f_{2}$ to take values on all of $\mathbb{R}$ and convexify the control set, we arrive at the exit time control problem of minimizing $T$ subject to

$$
\left\{\begin{array}{l}
\dot{x}=u_{1} \sqrt{|y|}, \quad \dot{y}=u_{2} \sqrt{|y|}, \quad\left(u_{1}, u_{2}\right)^{\prime} \in \overline{B_{1}(0)} \\
(x, y)^{\prime}(0)=p_{o}
\end{array}\right.
$$

and $(x, y)^{\prime}(T)=p_{f}$ for each singleton target $p_{f}$ and each choice of the initial position $p_{o}$, which is the RBP. Since the RBP dynamical law $f\left((x, y)^{\prime},\left(u_{1}, u_{2}\right)^{\prime}\right)=\sqrt{y}\left(u_{1}, u_{2}\right)^{\prime}$ is not Lipschitz, one cannot use the previously known uniqueness characterizations for viscosity solutions for exit time HJBE's to get a uniqueness characterization for the HJBE of the RBP. We sketch the proof that the RBP satisfies the hypotheses of Theorem 2.1.

The continuity of $T_{\overline{B_{1}(0)}, f}$ for each target $\left\{p_{f}\right\}$ follows from an elementary consideration of vertical and horizontal movements along RBP trajectories and the fact that RBP trajectories can be traveled in reverse. Set $\ell \equiv 1$ and $A=\overline{B_{1}(0)}$, and define $f_{n}$ by $f_{n}\left((x, y)^{\prime},\left(u_{x}, u_{y}\right)^{\prime}\right)=\left(y^{2}+1 / n\right)^{1 / 4}\left(u_{x}, u_{y}\right)^{\prime}$ for all $n \in \mathbb{N}$. These functions are Lipschitz, and $f_{n} \rightarrow f$ uniformly on compact sets. Since $\mathcal{R}_{f_{n}}(A) \equiv \mathbb{R}^{2}$, Condition 1 of the colue definition is satisfied. For each initial point $p \in \mathbb{R}^{2}$ and each input $\left(u_{x}^{\star}, u_{y}^{\star}\right)^{\prime} \in \mathcal{A}$, the corresponding trajectory $\left(\phi_{x}^{\star}, \phi_{y}^{\star}\right)^{\prime}$ for $f_{n}$ is also the trajectory for $f_{1}$ for the input

$$
\left[\left(\phi_{y}^{\star 2}(t)+1 / n\right)^{1 / 4} /\left(\phi_{y}^{\star 2}(t)+1\right)^{1 / 4}\right]\left(u_{x}^{\star}(t), u_{y}^{\star}(t)\right)^{\prime}
$$


Any trajectory $\left(\mu_{x}, \mu_{y}\right)^{\prime}$ for $f$ corresponding to an input $\left(\beta_{x}, \beta_{y}\right)^{\prime} \in \mathcal{A}$ is a trajectory for $f_{n}$ using the input

$$
\left[\sqrt{\left|\mu_{y}(t)\right|} /\left(\mu_{y}^{2}(t)+1 / n\right)^{1 / 4}\right]\left(\beta_{x}(t), \beta_{y}(t)\right)^{\prime}
$$

so Conditions 2 and 3 of the colue definition are also satisfied. One easily verifies that Condition 4 of the definition holds as well.

It remains to check that the RBP data is coercively transient. That each $\phi \in \operatorname{Traj}\left(p, f, \overline{B_{1}(0)},\left\{p_{f}\right\}\right)$ is coercifiable at $p=(x, y)^{\prime}$ when $y \neq 0$ follows from the fact that $f$ is Lipschitz away from $\left\{(x, y)^{\prime}: y=0\right\}$, so we assume $p=(x, 0)^{\prime} \neq p_{f}$ in the sequel. Fix $\alpha=\left(u_{x}, u_{y}\right) \in \mathcal{A}$ and $\phi=\left(\phi_{1}, \phi_{2}\right)^{\prime} \in \operatorname{Traj}_{\alpha}\left(p, f,\left\{p_{f}\right\}\right)$. Since $p \neq p_{f}$, and since the RBP dynamics does not allow horizontal movement along the $x$-axis, it follows that for some $s \in(0, \tau(\phi)), \phi(s)$ is not in the $x$-axis. We assume $\phi(s)=(\mu, \nu)^{\prime}$, with $\nu>0$. (The $\nu<0$ case is handled similarly.) Then $\phi\left\lceil[0, s]\right.$ is strictly below the $x$-axis on each interval in $\left\{\left(u_{j,-}, u_{j,+}\right)\right\}_{j \in S}$, where $S$ is at most countable. Set $\mathcal{D}_{\phi}=\bigcup_{j}\left(u_{j,-}, u_{j,+}\right)$. We reflect the subtrajectories $\phi\left\lceil\left(u_{j,-}, u_{j,+}\right)\right.$ over the $x$-axis.

Define $\tilde{\phi}=\left(\tilde{\phi}_{1}, \tilde{\phi}_{2}\right)^{\prime}$ and $\tilde{w}=\left(\tilde{u}_{x}, \tilde{u}_{y}\right)^{\prime}$ on $[0, s]$ by $\tilde{\phi}_{1}(t) \equiv \phi_{1}(t), \tilde{u}_{x}(t) \equiv u_{x}(t)$,

$$
\tilde{\phi}_{2}(t)=\left\{\begin{array}{ll}
+\phi_{2}(t), & t \notin \mathcal{D}_{\phi} \\
-\phi_{2}(t), & \text { otherwise }
\end{array} \quad \text { and } \quad \tilde{u}_{y}(t)= \begin{cases}+u_{y}(t), & t \notin \mathcal{D}_{\phi} \\
-u_{y}(t), & \text { otherwise }\end{cases}\right.
$$

Then, $\tilde{\phi}$ reaches $\phi(s)$ at some time $\tilde{s} \in(0, s]$ and lies completely in the closed upper half plane. Also, $(\tilde{\phi}, \tilde{w})$ is an RBP trajectory-control pair. Recall from [22] that the time optimal trajectories for joining points $P$ and $Q$ using the dynamics $\dot{x}=u_{x} \sqrt{y}, \dot{y}=u_{y} \sqrt{y}$ (with $\left(u_{x}, u_{y}\right)^{\prime} \in \overline{B_{1}(0)}, x \in \mathbb{R}$, and $y \geq 0$ ) are arcs of cycloids which pass from $P$ to $Q$ without hitting the $x$-axis in between. Since $p$ lies in the $x$-axis and $\phi(s)$ is above the axis, we can therefore replace $\tilde{\phi}\lceil[0, \tilde{s}]$ with a cylcoid arc $\hat{\phi}$ to get a trajectory that reaches $\phi(s)$ at some time $\hat{t} \in(0, \tilde{s}]$ and lies in the open upper half plane along $(0, \hat{t}]$. Moreover, since the RBP motion is Lipschitz on sets of the form $\{y \geq b\}$ for $b>0$, the RBP law is Lipschitz on $S_{n}$ for open sets $S_{n}$ containing Trace $\hat{\phi}\lceil[1 / n, \hat{t}]$ for $n$ large enough. Thus, we take $\psi=\hat{\phi}, t=s$, and $t^{\prime}=\hat{t}$ in the condition defining coercifiability of $\phi$ to satisfy the requirement. Applying Theorem 2.1, we obtain the following corollary:

Corollary 5.1. For each $p_{f} \in \mathbb{R}^{2}$, the value function of Sussmann's Reflected Brachystochrone Problem with target $\left\{p_{f}\right\}$ is the unique viscosity solution of the corresponding Bellman equation

$$
\sqrt{|y|}\left\|D v\left((x, y)^{\prime}\right)\right\|-1=0
$$

on $\mathbb{R}^{2} \backslash\left\{p_{f}\right\}$ in the class of functions $v \in C\left(\mathbb{R}^{2}\right)$ which are bounded below and which vanish at $p_{f}$.

\section{THREE EXTENSIONS OF THE MAIN RESUlT}

We close by showing how to relax the assumptions of Theorem 2.1. We first consider the case where all the hypotheses of the theorem are satisfied except that the pair $(f, \ell)$ is not coercively transient. We then consider the case where the colue is expansive but the other hypotheses of the theorem hold. Finally, we consider the case where $\ell$ is not bounded below by a positive constant, e.g., cases where $\ell(\cdot, a)$ is allowed to vanish at some points outside $\mathcal{T}$ for some choices of the input value $a$. This last case gives the uniqueness characterizations for the FP from [14] (e.g., Cor. 6.5 below). In the first two cases, we show that $v$ is the unique viscosity solution of the corresponding HJBE on $\mathcal{R} \backslash \mathcal{T}$ in a class of functions whose sub- or superdifferentials are locally bounded. In what follows, we continue to use the notation introduced in the proof of Theorem 2.1. Also, $C_{\text {locbsub }}(\mathcal{R})$ denotes the set of all continuous functions $u$ on $\mathcal{R}$ for which $\cup\left\{D^{-} u(x): x \in\left[\mathcal{R} \cap B_{r}(0)\right] \backslash \mathcal{T}\right\}$ is bounded for each $r>0$, and $C_{\text {locbsup }}(\mathcal{R})$ is the set of all continuous functions $w$ on $\mathcal{R}$ for which $\cup\left\{D^{+} w(x): x \in\left[\mathcal{R} \cap B_{r}(0)\right] \backslash \mathcal{T}\right\}$ is bounded for each $r>0$. The motivation for considering only semidifferentials for points outside $\mathcal{T}$ is that we are solving the HJBE $(2.3)$ on $\mathcal{R} \backslash \mathcal{T}$. Note that locally Lipschitz functions on $\mathcal{R}$ are in $C_{\text {locbsup }}(\mathcal{R}) \cap C_{\text {locbsub }}(\mathcal{R})$. 


\subsection{Uniqueness characterizations for nontransient colues}

Let the assumptions of Theorem 2.1 be satisfied except that $(f, \ell)$ may not be coercively transient, and assume $w \in C_{\text {locbsup }}(\mathcal{R})$ is a viscosity solution of the $\operatorname{HJBE}(2.3)$ on $\mathcal{R} \backslash \mathcal{T}$. Let $x \in \mathcal{R} \backslash \mathcal{T}, \alpha \in \mathcal{A}$, and $\phi \in \operatorname{Traj}_{\alpha}(x, f, \mathcal{T})$ be given. Set $S:=\{\phi(r): 0 \leq r \leq \tau(\phi)\}=$ Trace $\phi\lceil[0, \tau(\phi)]$, and pick $\varepsilon>0$ so that $S^{2 \varepsilon} \subseteq \mathcal{R}$. Set $\delta:=\frac{\varepsilon}{1+\tau(\phi)}$. We claim that there is an $N \in \mathbb{N}$ so that for all $n \geq N, w$ is a viscosity subsolution of

$$
\sup _{a \in A}\left[-f_{n}(p, a) \cdot D w(x)-\{\ell(p, a)+\delta\}\right]=0 \quad \text { on } \quad S^{\varepsilon} \backslash \mathcal{T} .
$$

Indeed, suppose this were false. Then for $n$ as large as desired, there would be values $x_{n} \in S^{\varepsilon} \backslash \mathcal{T}, a_{n} \in A$, and $p_{n} \in D^{+} w\left(x_{n}\right)$ so that

$$
-f_{n}\left(x_{n}, a_{n}\right) \cdot p_{n}-\ell\left(x_{n}, a_{n}\right) \geq \delta / 2 \quad \text { and } \quad-f\left(x_{n}, a_{n}\right) \cdot p_{n}-\ell\left(x_{n}, a_{n}\right) \leq \delta / 4,
$$

and then we arrive at a contradiction by subtracting these two inequalities and using the convergence on compact sets from the definition of colues and the boundedness of the $p_{n}$ 's.

By the colue assumption, we can also find a sequence $\beta_{n} \in \mathcal{A}$ so that $\left(\phi, \beta_{n}\right)$ is a trajectory-control pair for $f_{n}$ and $\left\|\beta_{n}\right\| \leq\|\alpha\|$ a.e. for all $n \in \mathbb{N}$. Since $f_{n}\left\lceil\left[S^{\varepsilon} \times A\right]\right.$ is Lipschitz on $S^{\varepsilon}$ for each $n \geq N$ and $\tau(\phi)<\infty$, Lemma 3.5 and the definition of $\delta$ give

$$
w(x) \leq \int_{0}^{\tau(\phi)} \ell\left(\phi(s), \beta_{n}(s)\right) \mathrm{d} s+\delta \tau(\phi)+w(\tau(\phi)) \leq \int_{0}^{\tau(\phi)} \ell(\phi(s), \alpha(s)) \mathrm{d} s+\varepsilon+w(\tau(\phi)),
$$

where the second inequality follows since $\ell \in C_{\mathrm{inc}}\left(\mathbb{R}^{N} \times A, \mathbb{R}_{+}\right)$. Also, in our proof of Theorem 2.1, the coercive transience hypothesis was only used to prove that any viscosity subsolution $w$ of the corresponding HJBE on $\mathcal{R} \backslash \mathcal{T}$ satisfying the condition $(S C)$ was pointwise at most $v$. Now assume $w$ also satisfies the condition $(S C)$. Then $w(\tau(\phi))=0$ in (6.1). Letting $\varepsilon \searrow 0$ and infimizing over $\operatorname{Traj}(x, f, A, \mathcal{T})$ in (6.1), we conclude as follows:

Theorem 6.1. Let $A$ be a compact normed vector space and $\mathcal{T} \subseteq \mathbb{R}^{N}$ be closed. Let $(f, \ell)$ be a colue, $T_{A, f}$ be continuous, and $\ell \geq 1$ on $\mathbb{R}^{N} \times A$. If $v_{f, \ell, A} \in C_{\text {locbsup }}(\mathcal{R})$, then it is the unique viscosity solution of the HJBE (2.3) in the class of functions $w \in C_{\text {locbsup }}(\mathcal{R})$ which satisfy $(S C)$.

Therefore, if $v_{f, \ell, A} \in C_{\text {locbsup }}(\mathcal{R})$, then it is possible to establish global existence and uniqueness of solutions for the HJBE (2.3) without checking for coercive transience, even if $f$ is non-Lipschitz. Here is an example of an eikonal equation from geometric optics for which this is done:

Example 6.1. Take $A=\overline{B_{1}(0)} \subseteq \mathbb{R}^{2}, N=2, \ell \equiv 1$, any closed target $\mathcal{T}$, and the non-Lipschitz dynamics $f(x, y, a, b)=\left(1+|y|^{1 / 2}\right)(a, b)$. (The argument we are about to give also applies if $\ell$ depends only on the state and $1 \leq \ell \leq M$ everywhere for some constant $M$.) While $f$ is non-Lipschitz, the existence of trajectories of $f$ can of course be established in the usual way using Schauder's fixed point theorem (cf. [2], p. 219). This gives the HJBE

$$
(1+\sqrt{|y|})\|D v(x, y)\|-1=0 .
$$

One approach to uniqueness of solutions for (6.2) is to rewrite (6.2) as

$$
\|D v(x, y)\|-(1+\sqrt{|y|})^{-1}=0,
$$

which is the HJBE for the exit time problem with the dynamics $\tilde{f}$ and Lagrangian $\tilde{\ell}$, where

$$
\tilde{f}(x, y, a, b)=(a, b) \in \overline{B_{1}(0)} \text { and } \quad \tilde{\ell}(x, y, a, b)=(1+\sqrt{|y|})^{-1} .
$$


Equation (6.3) is the eikonal equation for the propogation of light in a medium of speed $c(x, y)=1+|y|^{1 / 2}$. Let $\tilde{v}$ denote the exit time value function for the dynamics $\tilde{f}$, the Lagrangian $\tilde{\ell}$, and the target $\mathcal{T}$. While the transformation to the data (6.4) gives an exit time problem with Lipschitz dynamics, the well-known uniqueness characterizations for solutions of HJBE's cannot be applied to (6.3), since the Lagrangian $\tilde{\ell}$ is not bounded below by a positive constant. One might instead try to apply the methods from [14] for the case of exit time problems with vanishing Lagrangians and proper value functions to show that $\tilde{v} \in C\left(\mathbb{R}^{2}\right)$ is the unique viscosity solution of (6.3) on each sublevel set of $\tilde{v}$. The methods would apply since the sublevel sets of $\tilde{v}$ would be bounded. This would give the uniqueness characterization on all of $\mathbb{R}^{2}$ if $\tilde{v}$ were proper, since the union of the sublevel sets would be all of $\mathbb{R}^{2}$. However, $\tilde{v}$ is not necessarily proper, since $\mathcal{T}$ may be unbounded. The functions $f_{n}(x, y, a, b)=\left[1+\left(y^{2}+1 / n\right)^{1 / 4}\right](a, b)$ are Lipschitz, so the proof of Theorem 2.1 shows that $v_{f_{n}, \ell, A} \rightarrow v_{f, \ell, A}$ uniformly on compact subsets of $\mathbb{R}^{2}$. The continuity of $T_{A, f}$ follows from an elementary consideration of vertical and horizontal trajectories of $f$ and the strong reversibility of trajectories of $f$. The argument is similar to the one we used for the RBP dynamics. Therefore, we conclude from Lemma 3.4 that $v_{f, \ell, A}$ is a viscosity solution of $(6.2)$ on $\mathbb{R}^{2} \backslash \mathcal{T}$, and it is then immediate from the subsolution definition and (6.3) that $v_{f, \ell, A} \in C_{\text {locbsup }}\left(\mathbb{R}^{2}\right)$. We conclude from Theorem 6.1 that $v_{f, \ell, A}$ is the unique viscosity solution of $(6.2)$ on $\mathbb{R}^{2} \backslash \mathcal{T}$ in the class of functions $w \in C_{\text {locbsup }}\left(\mathbb{R}^{2}\right)$ that vanish on $\mathcal{T}$ and are bounded below.

The novelty of this argument is that it was not necessary to consider coercive transience as we did for the RBP. Also, the argument establishes uniqueness of solutions for an HJBE for Lipschitz dynamics and possibly unbounded targets by viewing it as the HJBE for a problem with non-Lipschitz dynamics.

\subsection{Uniqueness characterizations for expansive colues}

This subsection considers the case where all the hypotheses of Theorem 2.1 hold except that $(f, \ell)$ violates Condition 4 in the colue definition. For any set $B \subseteq \mathbb{R}^{N}$, the symbol STC(B) will mean that $B \subset \mathbb{R}^{N}$ and that for each $\varepsilon>0$, the set $B$ lies in the interior of the set of all points which can be brought to $B$ in time $<\varepsilon$ using the dynamics $f$ and some control in $\mathcal{A}$. For sufficient conditions for $\mathrm{STC}(\mathrm{B})$, see [20]. In the rest of this subsection, we assume $v_{f, \ell, A} \in C_{\text {locbsub }}\left(\mathcal{R}_{f}\right)$ and $\ell \geq 1$ on $\mathbb{R}^{N} \times A$.

We give conditions under which $v_{f, \ell, A}$ is the unique viscosity solution of (2.3) in the class of functions $w \in C_{\text {locbsub }}\left(\mathcal{R}_{f}\right)$ that satisfy the side condition (SC) and the following generalized properness condition:

(REG) For each $x \in \mathcal{R} \backslash \mathcal{T}$, there is a bounded open set $\Omega_{x} \subseteq \mathcal{R}$ containing $x$ and an $\omega_{o} \in \mathbb{R} \cup\{+\infty\}$ which are such that $\overline{\Omega_{x} \backslash \mathcal{T}} \subset \mathcal{R}$ and the following conditions hold:

$\left(\mathrm{REG}_{1}\right) \quad \lim _{\Omega_{x} \ni p \rightarrow x_{o}} w(p)=\omega_{o}$ for all $x_{o} \in \partial\left(\Omega_{x}\right)$.

$\left(\mathrm{REG}_{2}\right) \quad \begin{aligned} & \Omega_{x} \ni p \rightarrow x_{0} \\ & w(p)<\omega_{o} \text { for all } p \in \Omega_{x} .\end{aligned}$

$\left(\mathrm{REG}_{3}\right) \quad S T C\left(\left(\Omega_{x} \backslash \mathcal{T}\right)^{c}\right)$ holds for all $x$.

Functions satisfying (REG) are called $\mathcal{R}$-regular ( $c f$. [14]). Condition $\left(\mathrm{REG}_{3}\right)$ is redundant for cases where $T_{A, f}$ is continuous (but see Sect. 6.3 for results for cases where $T_{A, f}$ can be discontinuous, e.g., Fuller's example). For cases where $T_{A, f}$ is continuous, $\mathbb{R}^{N}=\mathcal{R}$, and $\mathcal{T}$ is bounded, this regularity condition is satisfied by proper continuous functions on $\mathbb{R}^{N}$ if we choose the $\Omega_{x}$ 's to be sublevel sets. (A function $w$ is said to be proper if $\lim _{\|x\| \rightarrow \infty} w(x)=+\infty$.) However, this condition can also be satisfied by functions which are not bounded below.

Let $w \in C_{\text {locbsub }}(\mathcal{R})$ be an $\mathcal{R}$-regular viscosity solution of the HJBE (2.3) on $\mathcal{R} \backslash \mathcal{T}$ for which $w \geq 0$ on $\mathcal{T}$. Let $x \in \mathcal{R} \backslash \mathcal{T}$, and choose an open set $\Omega_{x}$ containing $x$ which satisfies the requirements of the regularity condition (REG). Let $\varepsilon \in(0,1 / 2)$ be given, and set $\ell_{\varepsilon}:=\ell-\varepsilon$. There is an $N_{\varepsilon} \in \mathbb{N}$ so that if $n \geq N_{\varepsilon}$, then for all $p \in \Omega_{x} \backslash \mathcal{T}$ and $q \in D^{-} w(p)$, we can find an $\bar{a} \in \mathcal{M}(p, q)$ so that

$$
\begin{aligned}
0 \leq \sup _{a \in A}\{-f(p, a) \cdot q-\ell(p, a)\} & =-f(p, \bar{a}) \cdot q-\ell(p, \bar{a}) \\
& \leq-f_{n}(p, \bar{a}) \cdot q-[\ell(p, \bar{a})-\varepsilon] \leq \sup _{a \in A}\left\{-f_{n}(p, a) \cdot q-\ell_{\varepsilon}(p, a)\right\}
\end{aligned}
$$


(We require $\Omega_{x}$ to be bounded to justify the second inequality for cases where $f$ is expansive.) Via Lemma 3.3, for each $\varepsilon \in(0,1 / 2)$, we have

$$
w(p) \geq v_{f_{n(\varepsilon)}, \ell_{\varepsilon}, A}(p) \text { for all } p \in \Omega_{x} \text { for } n(\varepsilon) \text { large enough. }
$$

We establish that $w \geq v$ on $\Omega_{x}$ by checking that

$$
\lim _{\varepsilon \rightarrow 0} v_{f_{n(\varepsilon)}, \ell_{\varepsilon}, A}(p)=v(p) \text { for each fixed } p \in \Omega_{x}
$$

We will then conclude that $w \geq v$ on $\mathcal{R}$ (since the $\Omega_{x}$ 's engulf $\mathcal{R} \backslash \mathcal{T}$ ).

First note that $v_{f_{n(\varepsilon)}, \ell_{\varepsilon}, A}(p) \leq v_{f_{n(\varepsilon)}, \ell, A}(p)$ and recall from $(4.3)$ that $v_{f_{n(\varepsilon)}, \ell, A} \leq v$ on $\mathcal{R}$. Therefore, $v_{f_{n(\varepsilon)}, \ell_{\varepsilon}, A}(p) \leq v(p)$ for all $n$. Also, if we choose a sequence of inputs $\alpha \in \mathcal{A}$ and corresponding trajectories $\phi_{n} \in \operatorname{Traj}_{\alpha_{n}}\left(p, f_{n}, \mathcal{T}\right)$ so that

$$
v_{f_{n(\varepsilon)}, \ell_{\varepsilon}, A}(p)+1 \geq \int_{0}^{\tau\left(\phi_{n}\right)} \ell\left(\phi_{n}(s), \alpha_{n}(s)\right) \mathrm{d} s-\varepsilon \tau\left(\phi_{n}\right) \quad \text { for all } n,
$$

then

$$
v(p)+1+\varepsilon \tau\left(\phi_{n}\right) \geq \int_{0}^{\tau\left(\phi_{n}\right)} \ell\left(\phi_{n}(s), \alpha_{n}(s)\right) \mathrm{d} s \geq \tau\left(\phi_{n}\right),
$$

so $\tau\left(\phi_{n}\right) \leq(v(p)+1) /(1-\varepsilon)$. It follows that the infimization in the definition of the $v_{f_{n(\varepsilon)}, \ell_{\varepsilon}, A}(p)$ 's can be restricted to inputs with exit times which are at most $B_{p}:=[1 /(1-\varepsilon)](1+v(p))$. Recall that the part of the proof of Theorem 2.1 showing that $v_{f_{n}, \ell, A} \rightarrow v$ pointwise on $\mathcal{R}$ does not use the fact that the data are nonexpansive. Therefore,

$$
v(p)-v_{f_{n(\varepsilon)}, \ell_{\varepsilon}, A}(p) \leq v(p)-\left[v_{f_{n(\varepsilon)}, \ell, A}(p)-\varepsilon B_{p}\right]
$$

and the RHS is at most $\varepsilon(1+2(1+v(p)))$ for $n(\varepsilon)$ large enough, so we get (6.5). The part of the proof of Theorem 2.1 showing that $w(x) \leq v(x)$ on $\mathcal{R}$ makes no use of the nonexpansiveness assumption, nor does the part that shows $v$ is a viscosity solution of the HJBE (2.3) on $\mathcal{R} \backslash \mathcal{T}$. We therefore conclude as follows:

Theorem 6.2. Assume $A$ is a compact normed vector space, $\mathcal{T} \subseteq \mathbb{R}^{N}$ is closed, $(f, \ell)$ is a transient expansive colue, $T_{A, f}$ is continuous, and $\ell \geq 1$ on $\mathbb{R}^{N} \times A$. If $v_{f, \ell, A} \in \bar{C}_{\text {locbsub }}(\mathcal{R})$ is $\mathcal{R}$-regular, then it is the unique $\mathcal{R}$-regular viscosity solution of (2.3) in the class of functions in $C_{\text {locbsub }}(\mathcal{R})$ satisfying $(S C)$.

By the argument of Remark 2.5, Theorem 6.2 remains true if the control sets $A_{n}$ for the dynamics $f_{n}$ depend on $n$, as long as $A_{n} \downarrow A$ in the Haudorff sense, $\ell \equiv 1$, and $\mathcal{R}=\mathbb{R}^{N}$. As shown in Section 6.1, we can also replace the coercive transience assumption in Theorem 6.1 by the requirement that $v_{f, \ell, A}$ has locally bounded superdifferentials, in which case the conclusion of Theorem 6.2 becomes that $v_{f, \ell, A}$ is the unique $\mathcal{R}$-regular viscosity solution $w \in C_{\text {locbsub }}(\mathcal{R}) \cap C_{\text {locbsup }}(\mathcal{R})$ of the HJBE that satisfies (SC). Here is an example where the nonexpansiveness hypothesis in Theorem 2.1 and Theorem 6.1 is not satisfied but this variant of Theorem 6.2 can be used to establish uniqueness of solutions of the HJBE:

Example 6.2. Consider the following variant of Example 6.1. Take $A=\overline{B_{1}(0)} \subseteq \mathbb{R}^{2}, N=2, \ell \equiv 1$, the non-Lipschitz dynamics

$$
f(x, y, a, b)= \begin{cases}\left(1-|y|^{1 / 2}\right)(a, b), & |y| \leq 1 / 4 \\ (|y|+1 / 4)(a, b), & |y|>1 / 4\end{cases}
$$


and an arbitrary closed target $\mathcal{T}$ as the exit time problem data. This gives the HJBE

$$
\begin{cases}\left(1-|y|^{1 / 2}\right)\|D v(x, y)\|-1=0, & |y| \leq 1 / 4 \\ (|y|+1 / 4)\|D v(x, y)\|-1=0, & |y|>1 / 4\end{cases}
$$

This equation is of course equivalent to

$$
\begin{cases}\|D v(x, y)\|-[1-\sqrt{|y|}]^{-1}=0, & |y| \leq 1 / 4 \\ \|D v(x, y)\|-[|y|+1 / 4]^{-1}=0, & |y|>1 / 4\end{cases}
$$

which is the eikonal equation in geometric optics for light propogation in a medium with kinked speed

$$
c(x, y)= \begin{cases}1-\sqrt{|y|}, & |y| \leq 1 / 4 \\ |y|+1 / 4, & |y|>1 / 4\end{cases}
$$

As in Example 6.1 (6.7) is the HJBE for an exit time problem whose Lagrangian is not bounded below by a positive constant, so the known uniqueness characterizations for exit time HJBE's do not apply to (6.7). Using the approximating dynamics

$$
f_{n}(x, y, a, b)= \begin{cases}{\left[1-\left\{y^{2}+1 /(n+4)\right\}^{1 / 4}\right](a, b),} & |y| \leq 1 / 4 \\ {\left[|y|+1 / 4+\left(1 / 2-[1 / 16+1 /(n+4)]^{1 / 4}\right)\right](a, b),} & |y|>1 / 4\end{cases}
$$

with suitable control sets $A_{n} \downarrow A$ in the Hausdorff sense, the proof of Theorem 2.1 and the discussion in Remark 2.5 show that $v_{f, \ell, A}$ is a viscosity solution of the corresponding HJBE on $\mathbb{R}^{2} \backslash \mathcal{T}$ and is the uniform limit of the value functions $v_{n}$ for the data $f_{n}, A_{n}$, and $\ell \equiv 1$ on compact sets. However, one easily checks that the nonexpansiveness condition is not satisfied. On the other hand, standard arguments, e.g., Soravia's backward dynamic programming principle ( $c f$. [16], or Sect. III.2.3 of [2] with $\lambda=0$ and $t>0$ small), show that the value functions $v_{n}$ are bilateral viscosity supersolutions of the corresponding HJBE's on $\mathbb{R}^{2} \backslash \mathcal{T},{ }^{6}$ and then a one-sided variant of Lemma 3.4 ( $c f$. [2], pp. 35-6) establishes that $v_{f, \ell, A}$ is a bilateral supersolution of (6.6) on $\mathbb{R}^{2} \backslash \mathcal{T}$. Therefore, $v_{f, \ell, A} \in C_{\text {locbsub }}\left(\mathbb{R}^{2}\right)$. Also, the argument of Example 6.1 establishes that $v_{f, \ell, A}$ has locally bounded superdifferentials. We conclude from the proof of Theorem 6.2 that if $w \in C_{\text {locbsub }}\left(\mathbb{R}^{2}\right) \cap C_{\text {locbsup }}\left(\mathbb{R}^{2}\right)$ is an $\mathbb{R}^{2}$ regular viscosity solution of (6.6) on $\mathbb{R}^{2} \backslash \mathcal{T}$ which satisfies (SC), then $w \equiv v_{f, \ell, A}$. Since $\mathcal{T}$ may be unbounded, and since the Lagrangian $\tilde{\ell}$ for (6.7) is not bounded below by a positive constant, this result does not follow from the known results for exit time HJBE's (e.g., Cor. IV.4.3 of [2], and [15]). For the special case where $v_{f, \ell, A}$ is $\mathbb{R}^{2}$-regular, we also conclude that $v_{f, \ell, A}$ is the unique $\mathbb{R}^{2}$-regular viscosity solution $v \in C_{\text {locbsub }}\left(\mathbb{R}^{2}\right) \cap C_{\text {locbsup }}\left(\mathbb{R}^{2}\right)$ of (6.7) on $\mathbb{R}^{2} \backslash \mathcal{T}$ that satisfies (SC). The regularity condition on $v_{f, \ell, A}$ is satisfied if $\mathcal{T}$ is compact, since all trajectories of $f$ are trajectories of the Lipschitz dynamics $f_{1}$ with controls in $A_{1}$ and the minimal time function for $f_{1}$ is proper, which makes $v_{f, \ell, A}$ proper as well. (Indeed, for each $M>0$, our lemmas would give a bound $\tilde{K}$ for which $\left\|y_{x}^{f_{1}}(t, \alpha)\right\| \leq \tilde{K}$ for all $x \in \mathcal{T}$, inputs $\alpha$, and $t \in[0, M+1]$. If $v_{f_{1}, \ell, A_{1}}\left(x_{n}\right) \leq M<\infty$ with $\left\|x_{n}\right\| \rightarrow \infty$, then the strong reversibility of $f_{1}$ gives a sequence of trajectories $\left\{\phi_{j}\right\}$ for $f_{1}$ starting in $\mathcal{T}$ for which $\left\|\phi\left(t_{j}\right)\right\| \rightarrow \infty$ for some $t_{j} \in[0, M+1]$, a contradiction. Therefore, if $\mathcal{T}$ is bounded, then $v_{f_{1}, \ell, A_{1}}$ is proper, and then $v_{f, \ell, A}$ is also proper, since $v_{f, \ell, A} \geq v_{f_{1}, \ell, A_{1}}$ pointwise.)

\subsection{Uniqueness characterizations for problems with vanishing Lagrangians}

This subsection shows how to apply the methods of [14] to problems whose dynamics may be non-Lipschitz. We assume all the hypotheses of Theorem 2.1 are satisfied except that $\ell$ is not necessarily bounded below by

\footnotetext{
${ }^{6}$ Recall that if $F: \mathbb{R}^{N} \times \mathbb{R}^{N} \rightarrow \mathbb{R}$ is continuous, then a bilateral (viscosity) supersolution of $F(x, D u(x))=0$ on an open set $\mathcal{G}$ is defined to be a continuous function $u: \mathcal{G} \rightarrow \mathbb{R}$ for which $F\left(x_{o}, p\right)=0$ for all $x_{o} \in \mathcal{G}$ and $p \in D^{-} u\left(x_{o}\right)$.
} 
a positive constant. In particular, we allow cases where there are points $p \in \mathcal{R} \backslash \mathcal{T}$ with the property that $\ell(p, a)=0$ for some values of $a \in A$. We remark that even for problems where $f$ is Lipschitz, these cases are not covered by the previously known uniqueness characterizations for exit time HJBE's from [2,5], and [15]. We give conditions under which $v_{f, \ell, A}$ is the unique viscosity solution of $(2.3)$ in $C(\mathcal{R})$ which vanishes on $\mathcal{T}$ and satisfies the following weak version of (REG):

(QREG) For each $x \in \mathcal{R}$, there is a bounded open set $\Omega_{x} \subseteq \mathcal{R}$ containing $x$ and an $\omega_{o, x} \in \mathbb{R} \cup\{+\infty\}$ which are such that $\overline{\Omega_{x} \backslash \mathcal{T}} \subset \mathcal{R}$ and the following conditions hold:

$\left(\mathrm{QREG}_{1}\right) \quad \liminf _{\Omega_{x} \ni p \rightarrow x_{o}} w(p) \geq \omega_{o, x}$ for all $x_{o} \in \partial\left(\Omega_{x}\right) \backslash \mathcal{T}$.

$\left(\mathrm{QREG}_{2}\right) \quad w(x)<\omega_{o, x}$ for all $x$.

$\left(\mathrm{QREG}_{3}\right) \quad S T C\left(\left(\Omega_{x} \backslash \mathcal{T}\right)^{c}\right)$ holds for all $x$.

Condition $\left(\mathrm{QREG}_{3}\right)$ is redundant when $T_{A, f}$ is continuous. We include it here to simplify our discussion below of cases where $T_{A, f}$ is discontinuous. Functions $w$ satisfying (QREG) will be called $\mathcal{R}$ quasi-regular. This regularity condition is similar to the regularity condition (REG) of Section 6.2 , except that $\left(R E G_{1}, R E G_{2}\right)$ are relaxed. We relax $\left(R E G_{1}, R E G_{2}\right)$ so that we can apply the uniqueness results of this section to the FP ( $c f$. Cor. 6.5). In particular, we show that $v_{f, \ell, A}$ is the unique viscosity solution of (2.3) in a class of functions which includes functions which are not bounded below, and we use this result to obtain the uniqueness characterizations for the HJBE of the Fuller problem from [14]. We write $\omega_{o}$ instead of $\omega_{o, x}$ where this would not lead to confusion. We will require following:

(F1) For each $x \in \mathcal{R}, v(x) \leq v_{\infty}(x)$.

(F2) For each $x \in \mathcal{R} \backslash \mathcal{T}$ and $\alpha \in \mathcal{A}^{r}$, we have $\int_{0}^{t} \ell^{r}\left(y_{x}^{f_{1}, r}(s, \alpha), \alpha(s)\right) \mathrm{d} s>0$.

When Conditions (F1, F2) hold, we call $(f, \ell)$ Fuller-like.

Remark 6.3. We use the name Fuller-like since for the Fuller problem (cf. [14] and [25]), $v_{\infty}$ and $v$ coincide and (F2) is satisfied if we choose $f_{1}$ to be the dynamics $f\left((x, y)^{\prime}, a\right)=(y, a)^{\prime}$ of the original Fuller problem. (The Fuller Problem Lagrangian is $\ell\left((x, y)^{\prime}, a\right)=x^{2}$ and the control set is $A=[-1,+1]$.)

We now show how to modify the proof of Theorem 2.1 to get a uniqueness characterization for the HJBE (2.3) under the current hypotheses. Exactly as before, the $v_{n}$ 's are pointwise bounded and locally equicontinuous. In particular, we have $v_{n} \leq v$ on $\mathcal{R}$ for all $n$. Following the proof of Theorem 2.1, for each $p \in \mathcal{R} \backslash \mathcal{T}$, we choose a $\delta_{p}>0$ so that the $v_{n}$ 's are equicontinuous on $B_{\delta_{p}}(p) \subseteq \mathcal{R} \backslash \mathcal{T}$. For each $p \in \mathcal{R}$, we apply the AscoliArzela theorem on $\overline{B_{\delta_{p} / 2}(p)}$ and let $\bar{v}_{p}$ denote a uniform limit of a subsequence of the $v_{n}$ 's as in the proof of Theorem 2.1. To show $v$ is a viscosity solution of the HJBE on $\mathcal{R} \backslash \mathcal{T}$, we show that it is a viscosity solution of that equation on $B_{\delta_{p} / 2}(p) \subseteq \mathcal{R} \backslash \mathcal{T}$ for each $p \in \mathcal{R} \backslash \mathcal{T}$. This is valid since the test functions in the definition of viscosity solutions can be restricted to smaller neighborhoods. To do this, we prove that $v_{n}(x) \rightarrow v(x)$ for a fixed arbitrary $x \in B_{\delta_{p} / 2}(p)$ on compact sets and then argue exactly as in the proof of Theorem 2.1. Since $v_{n} \leq v$ on $\mathcal{R}$ for all $n$, it will follow that $v_{n}(x) \rightarrow v(x)$ along a subsequence if we show that for any $\varepsilon>0$, there is an $N_{\varepsilon} \in \mathbb{N}$ so that $v_{n}(x)+2 \varepsilon \geq v(x)$ for $n \geq N_{\varepsilon}$ (at least along a subsequence). This follows if the data are Fuller-like.

Indeed, pick trajectories $\phi_{n}$ for $f_{n}$ satisfying (4.8) in the proof of Theorem 2.1. We assume $\tau\left(\phi_{n}\right) \rightarrow \infty$ possibly by passing to a further subsequence. This is done since otherwise the proof would be exactly as before. Let $\left(\phi^{r}, \bar{\alpha}\right)$ be as in the proof of Theorem 2.1, and pick an $\alpha \in \mathcal{A}$ as in Lemma 3.8. By the Fuller-like assumption, we can choose a $W \in \mathbb{R}$ so that

$$
v(x)-\varepsilon \leq \int_{0}^{W} \ell\left(\phi^{r}(s), \alpha(s)\right) \mathrm{d} s
$$

This gives

$$
v_{n}(x)+\varepsilon \geq \int_{0}^{\tau\left(\phi_{n}\right) \wedge W} \ell\left(\phi_{n}(s), \alpha_{n}(s)\right) \mathrm{d} s \rightarrow \int_{0}^{W} \ell^{r}\left(\phi^{r}(s), \bar{\alpha}(s)\right) \mathrm{d} s=\int_{0}^{W} \ell\left(\phi^{r}(s), \alpha(s)\right) \mathrm{d} s \geq v(x)-\varepsilon
$$


by the arguments of Theorem 2.1. Therefore, for each $x \in B_{\delta_{p} / 2}(p)$,

$$
v_{n}(x) \rightarrow v(x)
$$

along a subsequence. Since $v_{n} \rightarrow v$ pointwise in $B_{\delta_{p} / 2}(p)$ and $v_{n} \rightarrow \bar{v}_{p}$ uniformly on $B_{\delta_{p} / 2}(p) \subseteq \mathcal{R} \backslash \mathcal{T}$ for any $p \in \mathcal{R}$, it follows that $v_{n} \rightarrow v$ uniformly on compact sets (along subsequences) in $\mathcal{R}$, so by Lemma 3.4 and the equicontinuity of the $v_{n}$ 's, $v$ is a viscosity solution of the HJBE on $\mathcal{R} \backslash \mathcal{T}$, as needed.

The proof that any other viscosity solution $w \in C(\mathcal{R})$ of $(2.3)$ on $\mathcal{R} \backslash \mathcal{T}$ which satisfies $(S C)$ is at least $v_{f, \ell, A}$ pointwise given in the proof of Theorem 2.1 also uses the fact that $\ell$ is bounded below by a positive constant. To show the minimality of $v_{f, \ell, A}$ when this lower bound condition fails, we prove uniqueness within a class of $\mathcal{R}$ quasi-regular functions. Let $w$ be any $\mathcal{R}$ quasi-regular viscosity solution of the HJBE (2.3) on $\mathcal{R} \backslash \mathcal{T}$ which vanishes on $\mathcal{T}$. By the proof of Theorem 2.1, we know that $w \leq v$ on $\mathcal{R}$. To prove the reverse inequality, fix $x \in \mathcal{R}$ and a corresponding $\Omega_{x}$ which satisfies the conditions in the definition (QREG), and let $\varepsilon>0$ be given. Then $\Omega_{x}$ is bounded. Using the nonexpansiveness assumption, it follows that $w$ is a viscosity supersolution of (4.1) for all large $n \in \mathbb{N}$. Using Lemma 3.6 and Assumption 2 from Definition 2.2, it follows that for $p \in \Omega_{x} \backslash \mathcal{T}, \delta \in(0,1 / 2]$, large $n$, and

$$
0 \leq t<\inf _{\alpha \in \mathcal{A}}\left\{s \geq 0: \operatorname{dist}\left(y_{p}^{f_{1}}(s, \alpha), \partial\left(\Omega_{x} \backslash \mathcal{T}\right)\right) \leq \delta \operatorname{dist}\left(p, \partial\left(\Omega_{x} \backslash \mathcal{T}\right)\right)\right\}
$$

we get

$$
w(p) \geq \inf _{\alpha \in \mathcal{A}}\left\{\int_{0}^{t} \ell\left(y_{p}^{f_{n}}(s, \alpha), \alpha(s)\right) \mathrm{d} s+w\left(y_{p}^{f_{n}}(t, \alpha)\right)\right\} .
$$

For each $n$, use the infimum definition to find $\alpha_{n} \in \mathcal{A}$ so that the RHS of (6.9) is at least

$$
-\frac{1}{n}+\int_{0}^{t} \ell\left(y_{p}^{f_{n}}\left(s, \alpha_{n}\right), \alpha_{n}(s)\right) \mathrm{d} s+w\left(y_{p}^{f_{n}}\left(t, \alpha_{n}\right)\right)
$$

Now we apply the weak- $\star$ convergence argument of the proof that $v_{n} \rightarrow v$ from Section 4 . In what follows, we do not relabel the convergent subsequences. Assume that

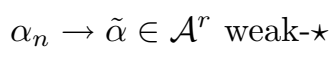

and that $\left(\beta_{n}, y_{x}^{f_{n}}\left(\cdot, \alpha_{n}\right)\right)$ is an input-trajectory pair for $f_{1}$ for all $n$ (cf. Def. 2.2). Assume that

$$
\beta_{n} \rightarrow \beta \in \mathcal{A}^{r} \text { weak- } \star
$$

Then $y_{x}^{f_{n}}\left(\cdot, \alpha_{n}\right)$ converges uniformly on compact sets to a relaxed trajectory $\phi^{r}$ for the relaxed control $\beta$ and $f_{1}^{r}$, and $\phi^{r}$ is also a relaxed trajectory for $\tilde{\alpha}$ (by exactly the argument we gave in the proof of Th. 2.1). Since the data are convex, $\phi^{r}$ is also a trajectory for $f$ for some input $\alpha \in \mathcal{A}$ (by Lem. 3.8). Letting $n \rightarrow \infty$ in $(6.10)$, and using the fact that $w$ is continuous at $\phi^{r}(t) \in \mathcal{R} \backslash \mathcal{T}$, we get

$$
w(p) \geq \inf \left\{\int_{0}^{t} \ell(\phi(s), \alpha(s)) \mathrm{d} s+w(\phi(t)): \alpha \in \mathcal{A}, \phi \in \operatorname{Traj}_{\alpha}\left(p, f, \mathbb{R}^{N}\right)\right\}
$$

for all $p \in \Omega_{x} \backslash \mathcal{T}$ and $t$ as above. That $w \geq v$ on $\Omega_{x}$ now follows from a variation of arguments from [14] which uses the fact that all trajectories of $f$ are trajectories of the Lipschitz dynamics $f_{1}$ but which does not use the continuity of $T_{A, f}$ or any other conditions from Definition 2.2 or Definition 2.3 ( $c f$. the Appendix below). Since $(f, \ell)$ is transient, we know $w \leq v$ on $\mathcal{R}$ as well, and this inequality holds trivially if $f$ is Lipschitz. When $f$ is Lipschitz, one shows that $v_{f, \ell, A}$ is a viscosity solution of (2.3) and that $v \leq w$ on $\mathcal{R}$ without requiring $T_{A, f}$ to be 
continuous ( $c f .[2])$. Since the results for Lipschitz data do not require the convexity of the sets $f(x, A) \times \ell(x, A)$, we conclude as follows:

Theorem 6.3. Assume $A$ is a compact normed vector space, $\mathcal{T} \subseteq \mathbb{R}^{N}$ is closed, and conditions (F1, F2) hold. Assume one of the following:

(i) $f$ is Lipschitz

(ii) $(f, \ell)$ is a transient colue, and $T_{A, f}$ is continuous.

If $v_{f, \ell, A}$ is $\mathcal{R}$ quasi-regular, then it is the unique $\mathcal{R}$ quasi-regular viscosity solution of (2.3) in $C(\mathcal{R})$ that vanishes on $\mathcal{T}$.

Remark 6.4. The proof of Theorem 6.3 only uses the nonexpansiveness assumption to establish that any $\mathcal{R}$ quasi-regular viscosity supersolution of (4.1) on $\mathcal{R} \backslash \mathcal{T}$ is bounded below by $v$ on $B$. Therefore, the theorem remains true if we relax the requirement that $(f, \ell)$ is a colue to the requirement that $(f, \ell)$ is an expansive colue and add local boundedness of the subdifferential to the regularity condition $(Q R E G)$. The argument is as in Section 6.2 and is obtained by replacing $\ell$ with $\ell-\varepsilon$ in (6.9) and (6.10) and then letting $\varepsilon \downarrow 0$ and $n \rightarrow \infty$. We leave the details to the reader.

Note that Theorem 6.3 concludes that $v_{f, \ell, A}$ is the unique viscosity solution of $(2.3)$ in a class of functions which includes functions which are not bounded below. The uniqueness results for the Fuller problem (FP) with exponent $q>1$ from [14] now follow by picking $f, \ell, A$, and $\mathcal{T}$ to be the FP data, so

$$
f\left((x, y)^{\prime}, a\right):=(y, a)^{\prime}, \quad A:=[-1,+1], \quad \ell\left((x, y)^{\prime}, a\right):=|x|^{q}, \quad \text { and } \quad \mathcal{T}:=\{\overrightarrow{0}\} \subset \mathbb{R}^{2} .
$$

We can take $f_{n} \equiv f$ since the FP dynamics is Lipschitz. For the same reason, we do not need $T_{A, f}$ to be continuous. The FP data give $\mathcal{R}=\mathbb{R}^{2}$. The fact that the FP value function is $\mathcal{R}$ quasi-regular follows from the following considerations. Let $S_{L}$ denote the open set bounded by the concatenation of the FP trajectory from $(0, L)^{\prime}$ to $(0,-L)^{\prime}$ using the constant control $-1 / 2$ followed by the trajectory back to $(0, L)^{\prime}$ using $a \equiv+1 / 2$ with the origin removed. We can then take $\Omega_{x}$ in (QREG) to be $S_{L}$ for $L=L(x)$ large enough, since $v$ is proper (cf. [25]). Since the FP value function is continuous, and since proper functions $w \in C\left(\mathbb{R}^{2}\right)$ satisfy (QREG) for the FP case (with the same choice of $\Omega_{x}$ 's), Theorem 6.3 and Remark 6.3 give the following result from [14]:

Corollary 6.5. For each $q>1$, the value function for the Fuller Problem with exponent $q$ is the unique viscosity solution of

$$
-y\left(D w\left((x, y)^{\prime}\right)\right)_{1}+\left|\left(D w\left((x, y)^{\prime}\right)\right)_{2}\right|-|x|^{q}=0
$$

on $\mathbb{R}^{2} \backslash\{0\}$ in the class of functions $w \in C\left(\mathbb{R}^{2}\right)$ satisfying $w(0)=0$ and $\lim _{\|x\| \rightarrow \infty} w(x)=+\infty$.

\section{ApPENDIX}

This appendix shows how to apply the methods of [14] for problems with singular Lagrangians to complete the proof of Theorem 6.3 above. In what follows $(A,\|\cdot\|)$ is a compact normed vector space and $\mathcal{T} \subseteq \mathbb{R}^{N}$ is closed. The notation will be as in the previous section. For $\Omega \subseteq \mathbb{R}^{N}$ fixed, we will set

$$
T_{\delta}(p):=\inf _{\alpha \in \mathcal{A}}\left\{t \geq 0: \operatorname{dist}\left(y_{p}^{f_{1}}(t, \alpha), \partial(\Omega \backslash \mathcal{T})\right) \leq \delta\right\}
$$

for each $\delta>0$ and $p \in \Omega$. We prove the following general result:

Proposition 7.1. Let $f \in C\left(\mathbb{R}^{N} \times A, \mathbb{R}^{N}\right)$, let $f_{1} \in C_{\text {lip }}\left(\mathbb{R}^{N} \times A, \mathbb{R}^{N}\right)$, let $\ell \in C_{\text {inc }}\left(\mathbb{R}^{N} \times A, \mathbb{R}_{+}\right)$, let $\Omega \subseteq \mathbb{R}^{N}$ be open and bounded, let $\omega_{o} \in \mathbb{R}$, and let $w \in C(\bar{\Omega})$. Assume the following:

1. For each $q \in \Omega, \alpha \in \mathcal{A}$, and $\phi \in \operatorname{Traj}_{\alpha}\left(q, f, \mathbb{R}^{N}\right)$, there is a $\beta \in \mathcal{A}$ so that $\phi \in \operatorname{Traj}_{\beta}\left(q, f_{1}, \mathbb{R}^{N}\right)$ and so that $\|\beta\| \leq\|\alpha\|$ a.e. 
2. For each $q \in \Omega \cap \mathcal{T}$, we have $w(q) \geq 0$.

3. $\inf \{w(q): q \in[\partial \Omega] \backslash \mathcal{T}\} \geq \omega_{o}$.

4. If $q \in \Omega \backslash \mathcal{T}$, then $w(q) \geq \inf \left\{\int_{0}^{t} \ell(\phi(s), \alpha(s)) \mathrm{d} s+w(\phi(t)): \alpha \in \mathcal{A}, \phi \in \operatorname{Traj}_{\alpha}\left(q, f, \mathbb{R}^{N}\right)\right\}$ for all $0 \leq$ $t<T_{\delta}(q)$ and $0<\delta \leq 1 / 4 \operatorname{dist}(q, \partial(\Omega \backslash \mathcal{T}))$.

5. For each $q \in \Omega \backslash \mathcal{T}, \alpha \in \mathcal{A}^{r}$, and $t \in(0,+\infty]$, we have $\int_{0}^{t} \ell^{r}\left(y_{q}^{f_{1}, r}(s, \alpha), \alpha(s)\right) \mathrm{d} s \in(0, \infty]$.

6. $\operatorname{STC}\left((\Omega \backslash \mathcal{T})^{c}\right)$.

Then $w(x) \geq v_{f, \ell, A}(x)$ for all $x \in\left\{p \in \Omega: w(p)<\omega_{o}\right\}$.

Note that this result does not assume that $w$ is a viscosity solution of any HJBE's. If the hypotheses of Proposition 7.1 are satisfied, then it must be the case that $\left\{x \in \Omega: w(x)<\omega_{o}\right\} \subseteq \mathcal{R}$ (since $v_{f, \ell, A} \equiv+\infty$ on $\left.\mathbb{R}^{N} \backslash \mathcal{R}\right)$. Also, the proposition remains true if instead of assuming that $w \in C(\bar{\Omega})$, we assume that $w$ is lower semicontinuous on $\bar{\Omega}$. Notice that Proposition 7.1 does not require $\partial \mathcal{T}$ to be compact, nor do we require that $\mathcal{T} \subseteq \Omega$. However, the proof will show that $\bar{\Omega} \cap \mathcal{T} \neq \emptyset$. Once this proposition is shown, the proof of Theorem 6.3 will be complete, since we can use the condition $\mathcal{R} \backslash \mathcal{T}=\cup\left\{\Omega_{x} \backslash \mathcal{T}: x \in \mathcal{R} \backslash \mathcal{T}\right\}$ from the assumption (QREG) to conclude that $w(x) \geq v_{f, \ell, A}(x)$ for all $x \in \mathcal{R} \backslash \mathcal{T}$, hence for all $x \in \mathcal{R}$ (since $w \equiv v_{f, \ell, A}$ on $\mathcal{T}$ ).

The proof of Proposition 7.1 is a generalization of arguments in [14]. Let $x \in \Omega \backslash \mathcal{T}$ be such that $w(x)<\omega_{o}$, and let $\varepsilon \in\left(0,\left[\omega_{0}-w(x)\right] \wedge 1\right)$ be given. For each $p \in \Omega \backslash \mathcal{T}$ and $\phi \in \operatorname{Traj}\left(p, f, A, \mathbb{R}^{N}\right)$, we set

$$
\hat{\tau}(\phi)=\inf \{t \geq 0: \phi(t) \in \partial(\Omega \backslash \mathcal{T})\}
$$

We construct an $\hat{\alpha} \in \mathcal{A}$ and a $\hat{\phi} \in \operatorname{Traj}_{\hat{\alpha}}(x, f, \partial(\Omega \backslash \mathcal{T}))$ so that

$$
w(x) \geq \int_{0}^{\hat{\tau}(\hat{\phi})} \ell(\hat{\phi}(s), \hat{\alpha}(s)) \mathrm{d} s+\lambda_{x}(\hat{\phi})-\varepsilon
$$

where

$$
\lambda_{x}(\phi):= \begin{cases}0, & \hat{\tau}(\phi)=\tau(\phi) \\ \frac{1}{2}\left(w(x)+\omega_{o}\right), & \hat{\tau}(\phi) \neq \tau(\phi) .\end{cases}
$$

To do this, we apply Assumption 4 repeatedly to a sequence of points $x_{1}, x_{2}, \ldots$ in $\Omega \backslash \mathcal{T}$. We assume in each application that $\delta$ can be taken to be 1 . The general case then follows from the proof we now give by replacing $T_{1 / k}\left(x_{k}\right)$ with $T_{\delta_{k}}\left(x_{k}\right)$ for a suitable sequence $\delta_{k} \downarrow 0$. We define $\left\{x_{k}\right\}$ recursively as follows. Set $x_{1}=x$, $\tau_{1}:=T_{1}(x)$ if $T_{1}(x)<\infty$, and $\tau_{1}=10$ if $T_{1}(x)=+\infty$. Using Assumption 4, we can find an $\alpha_{1} \in \mathcal{A}$ and a $\phi_{1} \in \operatorname{Traj}_{\alpha_{1}}\left(x, f, \mathbb{R}^{N}\right)$ for which

$$
w\left(x_{1}\right) \geq \int_{0}^{\tau_{1}} \ell\left(\phi_{1}(s), \alpha_{1}(s)\right) \mathrm{d} s+w\left(\phi_{1}\left(\tau_{1}\right)\right)-\varepsilon / 4
$$

The existence of such a $\phi_{1}$ is immediate from Assumption 4 if $T_{1}\left(x_{1}\right)=+\infty$, and it follows trivially if $T_{1}\left(x_{1}\right)=0$. Otherwise, the estimates of Lemma 3.1 give $0<T_{1+\mu}(x)<T_{1}(x)<+\infty$ for some $\mu>0(\operatorname{since} \operatorname{dist}(x, \partial(\Omega \backslash \mathcal{T}))>$ 1) and we can take $\mu$ as small as desired, so we can take $\tau_{1}=T_{1+\mu}$ instead. To simplify the notation, we always assume that we can put $t=T_{1 / k}\left(x_{k}\right)$ in Assumption 4. By construction, $\phi_{1}\left(\tau_{1}\right) \in \Omega \backslash \mathcal{T}$. By induction, we define $x_{k}=\phi_{k-1}\left(\tau_{k-1}\right) \in \Omega \backslash \mathcal{T}$, where we set $\tau_{k}=T_{1 / k}\left(x_{k}\right)$ if $T_{1 / k}<\infty$ and $\tau_{k}=10^{k}$ if $T_{1 / k}=+\infty$.

Since $x_{k} \in \Omega \backslash \mathcal{T}$, we can reapply Assumption 4 to find $\alpha_{k} \in \mathcal{A}$ and $\phi_{k} \in \operatorname{Traj}_{\alpha_{k}}\left(x_{k}, f, \mathbb{R}^{N}\right)$ such that

$$
w\left(x_{k}\right) \geq \int_{0}^{\tau_{k}} \ell\left(\phi_{k}(s), \alpha_{k}(s)\right) \mathrm{d} s+w\left(\phi_{k}\left(\tau_{k}\right)\right)-2^{-(k+1)} \varepsilon \text { for all } k \in \mathbb{N} .
$$


We also set $\sigma_{0}=0, \sigma_{k}:=\tau_{1}+\ldots+\tau_{k}$ for $k \geq 1, \bar{\sigma}=\lim \sup \sigma_{k}$, and, for an arbitrary $\bar{a} \in A$,

$$
\bar{\alpha}(s):= \begin{cases}\alpha_{1}(s) & \text { if } 0 \leq s<\sigma_{1} \\ \alpha_{2}\left(s-\sigma_{1}\right) & \text { if } \sigma_{1} \leq s<\sigma_{2}, \\ \vdots & \\ \alpha_{k}\left(s-\sigma_{k-1}\right) & \text { if } \sigma_{k-1} \leq s<\sigma_{k} \\ \vdots & \text { if } \bar{\sigma} \leq s, \\ \bar{a} & \end{cases}
$$

with the last line making sense for $\bar{\sigma}<+\infty$. Choose $\bar{\phi} \in \operatorname{Traj}_{\bar{\alpha}}\left(x, f, \mathbb{R}^{N}\right)$ such that $\bar{\phi}(s)=\phi_{k}\left(s-\sigma_{k}\right)$ for all $k$ and all $s \in\left[\sigma_{k-1}, \sigma_{k}\right]$. The existence of such a $\bar{\phi}$ is immediate if $\bar{\sigma}=+\infty$. If $\bar{\sigma}<+\infty$, use the boundedness of the $x_{k}$ 's to find a point $x^{+} \in \bar{\Omega}$ such that $\phi_{k}\left(\tau_{k}\right) \rightarrow x^{+}$along a subsequence, pick $\phi^{+} \in \operatorname{Traj}_{\alpha \equiv \bar{a}}\left(x^{+}, f, \mathbb{R}^{N}\right)$, and define $\bar{\phi}$ by $\bar{\phi}(s)=\phi_{k}\left(s-\sigma_{k}\right)$ for all $k$ and all $s \in\left[\sigma_{k-1}, \sigma_{k}\right]$ and $\bar{\phi}(s)=\phi^{+}(s-\bar{\sigma})$ for $s \geq \bar{\sigma}$. Standard ODE arguments then establish that

$$
\bar{\phi}(t)=x+\int_{0}^{t} f(\bar{\phi}(s), \bar{\alpha}(s)) \mathrm{d} s \quad \forall t \geq 0 .
$$

By Assumption 1, it follows that there exists a $\bar{\beta} \in \mathcal{A}$ such that $\bar{\phi}(s)=y_{x}^{f_{1}}(s, \bar{\beta})$ for all $s \geq 0$ and such that $\|\bar{\beta}\| \leq\|\bar{\alpha}\|$ a.e. Also, $\bar{\phi}$ stays in $\Omega \backslash \mathcal{T}$ for $s<\bar{\sigma}$. Since

$$
\int_{0}^{\tau_{k}} \ell\left(\phi_{k}(s), \alpha_{k}(s)\right) \mathrm{d} s=\int_{\sigma_{k-1}}^{\sigma_{k}} \ell(\bar{\phi}(s), \bar{\alpha}(s)) \mathrm{d} s>0 \text { for all } k,
$$

we conclude that

$$
\begin{aligned}
w(x) & \geq \int_{0}^{\sigma_{1}} \ell(\bar{\phi}(s), \bar{\alpha}(s)) \mathrm{d} s+w\left(x_{2}\right)-\frac{\varepsilon}{4} \geq \int_{0}^{\sigma_{2}} \ell(\bar{\phi}(s), \bar{\alpha}(s)) \mathrm{d} s+w\left(x_{3}\right)-\varepsilon\left(\frac{1}{4}+\frac{1}{8}\right) \\
& \geq \ldots \geq \sum_{m=1}^{k-1} \int_{\sigma_{m-1}}^{\sigma_{m}} \ell(\bar{\phi}(s), \bar{\alpha}(s)) \mathrm{d} s+w\left(x_{k}\right)-\frac{\varepsilon}{2}\left(1-\frac{1}{2^{k-1}}\right) \forall k .
\end{aligned}
$$

By the boundedness of $\Omega,\left\{x_{k}\right\}$ is bounded and therefore clusters. Let $\bar{x}$ be a cluster point of the $x_{k}$ 's, and assume that $x_{k} \rightarrow \bar{x}$ (by passing to a subsequence without relabeling). Then $\bar{x} \in \overline{\Omega \backslash \mathcal{T}}$. By our choice of $\varepsilon$, it follows that $\bar{x}$ is not in $\partial \Omega$. We will need the following minimality property of $\bar{x}$ :

Claim 7.2. In the above notation, $\bar{\tau}:=\inf \left\{\hat{\tau}(\phi): \phi \in \operatorname{Traj}\left(\bar{x}, f_{1}, A, \mathbb{R}^{N}\right)\right\} \leq \limsup \tau_{k}$.

Proof. First assume $\bar{\tau}<\infty$. Let $\delta>0$ be given, and suppose that, for $k$ as large as desired, we had $\tau_{k}<\bar{\tau}-\delta$. Passing to a subsequence, we assume that $\tau_{k} \rightarrow z \in[0, \bar{\tau}-\delta]$. There would then exist sequences $\tilde{\tau}_{k} \rightarrow z$, $\mu_{k} \in \operatorname{Traj}\left(x_{k}, f, A, \mathbb{R}^{N}\right)$, and $u_{k} \in \mathcal{A}$ so that $\mu_{k}(s)=y_{x_{k}}^{f_{1}}\left(s, u_{k}\right)$ for all $k$ and a relaxed control $u \in \mathcal{A}^{r}$ such that

$$
\operatorname{dist}\left(y_{\bar{x}}^{f_{1}, r}(z, u), \partial(\Omega \backslash \mathcal{T})\right) \leftarrow \operatorname{dist}\left(\mu_{k}\left(\tilde{\tau}_{k}\right), \partial(\Omega \backslash \mathcal{T})\right) \leq \frac{1}{k} \rightarrow 0 \text { as } k \rightarrow+\infty .
$$

The existence of the $u_{k}$ and $\mu_{k}$ 's follows from the definition of the infima $\tau_{k}$ and Assumption 1. To check (7.3), apply the compactness lemma of Section 3 to the sequence $u_{k}$, the interval $[0, \bar{\tau}-\delta]$, and the Lipschitz dynamics $f_{1}$ to get a weak- $\star$ limit $u \in \mathcal{A}^{r}$ such that

$$
\left\|y_{\bar{x}}^{f_{1}, r}(z, u)-\mu_{k}\left(\tilde{\tau}_{k}\right)\right\| \leq\left\|y_{\bar{x}}^{f_{1}, r}(z, u)-y_{\bar{x}}^{f_{1}, r}\left(\tilde{\tau}_{k}, u\right)\right\|+\left\|y_{\bar{x}}^{f_{1}, r}\left(\tilde{\tau}_{k}, u\right)-y_{x_{k}}^{f_{1}}\left(\tilde{\tau}_{k}, u_{k}\right)\right\| \rightarrow 0 .
$$


The result $(7.3)$ now follows since $\operatorname{dist}(\cdot, \partial(\Omega \backslash \mathcal{T}))$ is continuous.

The trajectories $y_{\bar{x}}^{f_{1}}\left(\cdot, u_{k}\right)$ converge uniformly to $y_{\bar{x}}^{f_{1}, r}(\cdot, u)$ on $[0, z+1]$ (by the Compactness lemma), and (7.3) gives

$$
y_{\bar{x}}^{f_{1}}\left(\tilde{\tau}_{k}, u_{k}\right) \quad \rightarrow \quad y_{\bar{x}}^{f_{1}, r}(z, u) \quad \in \partial(\Omega \backslash \mathcal{T}) \quad \text { as } \quad k \rightarrow \infty
$$

Therefore, for large $k$, we know that $y_{\bar{x}}^{f_{1}}\left(\tilde{\tau}_{k}, u_{k}\right)$ lies in $\Omega \backslash \mathcal{T}$ (since we may suppose that $\tilde{\tau}_{k} \leq \bar{\tau}-\delta$ for all $k$ ) and can be brought to $\partial(\Omega \backslash \mathcal{T})$ by the dynamics $f_{1}$ and some control $\tilde{u}$ in time less than $\delta / 2$ (by Assumpt. 1 , Assumpt. 6, and (7.4)). If we concatenate a control $u_{k}$ for such a $k$ and a corresponding control $\tilde{u}$, we get a trajectory for $f_{1}$ which brings $\bar{x}$ to $\partial(\Omega \backslash \mathcal{T})$ in time $\leq \bar{\tau}-\delta / 4$, which stands in contradiction to the definition of $\bar{\tau}$. We conclude that $\tau_{k} \geq \bar{\tau}-\delta$ for $k$ large enough along a subsequence, which establishes the result if $\bar{\tau}<\infty$, since $\delta>0$ was arbitrary. If $\bar{\tau}=+\infty$, then replace $\bar{\tau}-\delta$ in the preceding argument with any positive number to get the same result.

Passing to a further subsequence without relabeling, define $l \geq \bar{\tau}$ by $\tau_{k} \uparrow l \in[0,+\infty]$. By the estimates of Lemma 3.1, we know that $\bar{\tau}=0$ iff $\bar{x} \in \partial(\Omega \backslash \mathcal{T})$. Indeed, if $\bar{x} \notin \partial(\Omega \backslash \mathcal{T})$, then $\bar{x} \in \Omega \backslash \mathcal{T}$ (since $x_{k} \in \Omega \backslash \mathcal{T}$ for all $k$ ), so $B_{\mu}(\bar{x}) \subseteq \Omega \backslash \mathcal{T}$ for some $\mu>0$. By Result 2 of Lemma 3.1, there is a $\gamma>0$ so that all trajectories for $f_{1}$ which start at $\bar{x}$ and run for time $\leq \gamma$ stay in $B_{\mu}(\bar{x})$, so $\bar{\tau} \geq \gamma>0$. The converse is trivial. We use the following consequence of Claim 7.2:

Claim 7.3. With the above notation, we have $\bar{x} \in \partial \mathcal{T}$.

Proof. Let $M \in(0, l)$, and set $\beta_{k}\left(s-\sigma_{k-1}\right) \equiv \bar{\beta}(s)$, so $\phi_{k}(s)=y_{x_{k}}^{f_{1}}\left(s, \beta_{k}\right)$ for all $s \in\left(0, \tau_{k}\right)$ and all $k$. Let $\tilde{\beta}$ be a weak- $\star$ limit of a subsequence of these $\beta_{k}$ 's on $[0, M]$, which we assume to be the sequence itself for the sake of brevity. From the definition of the $\sigma_{k}$ 's, we also know that as $k \rightarrow \infty$, we have

$$
\begin{aligned}
0 & \leftarrow \int_{\sigma_{k-1}}^{\sigma_{k} \wedge\left\{\sigma_{k-1}+M\right\}} \ell(\bar{\phi}(s), \bar{\alpha}(s)) \mathrm{d} s \geq \int_{\sigma_{k-1}}^{\sigma_{k} \wedge\left\{\sigma_{k-1}+M\right\}} \ell\left(y_{x}^{f_{1}}(s, \bar{\beta}), \bar{\beta}(s)\right) \mathrm{d} s \\
& =\int_{0}^{\tau_{k} \wedge M} \ell\left(y_{x_{k}}^{f_{1}}\left(s, \beta_{k}\right), \beta_{k}(s)\right) \mathrm{d} s \rightarrow \int_{0}^{l \wedge M} \ell^{r}\left(y_{\bar{x}}^{f_{1}, r}(s, \tilde{\beta}), \tilde{\beta}(s)\right) \mathrm{d} s \geq 0 .
\end{aligned}
$$

The left arrow is by the divergence test applied to the partial sums in (7.2), since $w$ is bounded below on $\bar{\Omega}$. The first inequality follows since $\ell \in C_{\text {inc }}\left(\mathbb{R}^{N} \times A, \mathbb{R}_{+}\right)$. To justify the right arrow, first apply the compactness lemma (cf. Lem. 3.7) to get a $\tilde{\beta} \in \mathcal{A}^{r}$ such that

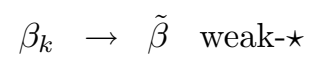

and such that

$$
y_{x_{k}}^{f_{1}, r}\left(\cdot, \beta_{k}\right) \rightarrow y_{\bar{x}}^{f_{1}, r}(\cdot, \tilde{\beta}) \quad \text { uniformly } \quad \text { on } \quad[0, M]
$$

Let $\beta_{k, s}(\cdot)$ (resp., $\left.\tilde{\beta}_{s}(\cdot)\right)$ denote the Radon measures $\beta_{k}(s)$ (resp., $\tilde{\beta}(s)$ ) for each $k$ and $s$. Then,

$$
\begin{aligned}
\left|\int_{0}^{M}\left[\ell^{r}\left(y_{\bar{x}}^{f_{1}, r}(s, \tilde{\beta}), \tilde{\beta}(s)\right)-\ell^{r}\left(y_{x_{k}}^{f_{1}, r}\left(s, \beta_{k}\right), \beta_{k}(s)\right)\right] \mathrm{d} s\right| \leq \mid \int_{0}^{M} \int_{A} \ell\left(y_{\bar{x}}^{f_{1}, r}(s, \tilde{\beta}), a\right) \mathrm{d} \tilde{\beta}_{s}(a) \mathrm{d} s \\
-\int_{0}^{M} \int_{A} \ell\left(y_{\bar{x}}^{f_{1}, r}(s, \tilde{\beta}), a\right) \mathrm{d} \beta_{k, s}(a) \mathrm{d} s|+| \int_{0}^{M} \int_{A}\left[\ell\left(y_{\bar{x}}^{f_{1}, r}(s, \tilde{\beta}), a\right)-\ell\left(y_{x_{k}}^{f_{1}, r}\left(s, \beta_{k}\right), a\right)\right] \mathrm{d} \beta_{k, s}(a) \mathrm{d} s \mid .
\end{aligned}
$$


The first RHS term tends to 0 as $k \rightarrow \infty$ because $\beta_{k} \rightarrow \tilde{\beta}$ weak-star on $[0, M]$ and because we can set $(h(s))(a):=\ell\left(y_{\bar{x}}^{f_{1}, r}(s, \tilde{\beta}), a\right)$ in $(2.1)$. (Notice that we are using the fact that $\ell$ is continuous in the control set value.) The second RHS term tends to 0 by (7.6) and the compactness of $A$. (Notice that there is a modulus $\omega_{\Omega}$ so that $|\ell(p, a)-\ell(q, a)| \leq \omega_{\Omega}(\|p-q\|)$ for all $p, q \in \bar{\Omega}$ and $a \in A$, since $A$ is compact.) This justifies the right arrow in (7.5) if $l>M$.

If $\int_{0}^{\bar{\tau}} \ell^{r}\left(y_{\bar{x}}^{f_{1}, r}(s, \tilde{\beta}), \tilde{\beta}(s)\right) \mathrm{d} s>0$, then $\int_{0}^{G} \ell^{r}\left(y_{\bar{x}}^{f_{1}, r}(s, \tilde{\beta}), \tilde{\beta}(s)\right) \mathrm{d} s>0$ for some $G \in(0, \bar{\tau})$. Since $l \geq \bar{\tau}(c f$. Claim 7.2), we would reach a contradiction by putting $M=G$ in (7.5). Therefore, by Assumption $5, \bar{\tau}=0$, or $\bar{x}$ is not in $\Omega \backslash \mathcal{T}$. If $\bar{\tau}=0$, then $\bar{x} \in \partial(\Omega \backslash \mathcal{T})$, as explained above. Assume $\bar{x} \notin \Omega \backslash \mathcal{T}$. Since $\bar{x} \in \overline{\Omega \backslash \mathcal{T}}$ by construction, we again conclude that $\bar{x} \in \partial(\Omega \backslash \mathcal{T})$. Since we already established that $\bar{x} \notin \partial \Omega$, the result follows.

We can therefore find an $\bar{n} \in \mathbb{N}$ such that for each $k \geq \bar{n}$, there exists a $\gamma_{k} \in \mathcal{A}$ and a trajectory $\lambda_{k} \in$ $\operatorname{Traj}_{\gamma_{k}}\left(x_{k}, f, \partial \mathcal{T}\right)$ which drives $x_{k}$ to a point $\tilde{x} \in \partial(\Omega \backslash \mathcal{T})$ (depending on $k$ ) and which is such that

$$
\int_{0}^{\hat{\tau}\left(\lambda_{k}\right)} \ell\left(\lambda_{k}(s), \gamma_{k}(s)\right) \mathrm{d} s<\varepsilon / 4
$$

To see why, let $F$ be a bounded set containing $\left\{y_{x_{k}}^{f_{1}}(s, \beta): \beta \in \mathcal{A}, 0 \leq s \leq 1, k \in \mathbb{N}\right\}$. Such a set exists by the second estimate of Lemma 3.1 and the boundedness of the $x_{k}$ 's. Recall that we are assuming that all trajectories of $f$ are also trajectories of $f_{1}$. Set $\bar{\kappa}=\sup \{\ell\lceil[F \times A]\}+1$. This is finite since $A$ is compact and $\ell$ is continuous. By Assumption 6 and the fact that $\left\{x_{k}\right\}$ converges to a point in $\bar{x} \in \partial(\Omega \backslash \mathcal{T})$, we can find a $\bar{n} \in \mathbb{N}$ so that if $k>\bar{n}$, then $x_{k}$ can be brought to a point $\tilde{x} \in \partial(\Omega \backslash \mathcal{T})$ (which depends on $k$ ) in time $<\varepsilon /(4 \bar{\kappa})$ by some trajectory $\lambda_{k}$ for $f$. This establishes (7.7).

For $k>\bar{n}$ in $\mathbb{N}$, our construction (7.2) therefore gives

$$
w(x) \geq \int_{0}^{\sigma_{k-1}} \ell(\bar{\phi}(s), \bar{\alpha}(s)) \mathrm{d} s+w\left(x_{k}\right)+\int_{0}^{\hat{\tau}\left(\lambda_{k}\right)} \ell\left(\lambda_{k}(s), \gamma_{k}(s)\right) \mathrm{d} s-\varepsilon / 2\left(1-2^{-(k-1)}+1 / 2\right) .
$$

There is a $\overline{\bar{n}} \in \mathbb{N}$ so that if $k \geq \overline{\bar{n}}$ and if $\tilde{x} \in \mathcal{T}$, then $w\left(x_{k}\right)+\varepsilon / 4>w(\tilde{x})$. To see why, first recall that $w \in C(\bar{\Omega})$, and note that the running times $\hat{\tau}\left(\lambda_{k}\right)$ can be taken as small as desired by taking $k$ large (by the argument of the preceding paragraph). By choosing small enough running times for the paths from $x_{k}$ to $\tilde{x} \in \partial(\Omega \backslash \mathcal{T})$, we can use the first estimate from Lemma 3.1 to ensure that $\left\|x_{k}-\tilde{x}\right\|<\delta$, where $\delta$ is chosen so that

$$
|w(p)-w(a)|<\varepsilon / 4 \text { for all } a \in \mathcal{T} \cap \bar{\Omega} \text { and } p \in[\mathcal{T} \cap \bar{\Omega}]^{\delta} \cap \bar{\Omega} .
$$

Such a $\delta$ exists since $w$ is continuous on the compact set $\bar{\Omega}$. The estimate now follows by choosing $a=\tilde{x}$ and $p=x_{k}$.

If on the other hand the index $k$ is such that $\tilde{x} \in \partial \Omega \backslash \mathcal{T}$, then the assumption that $w(x)<\omega_{o}$, Assumption 3 , and arguments similar to the ones we gave in the previous paragraph guarantee that $w\left(x_{k}\right) \geq \frac{1}{2}\left[w(x)+\omega_{o}\right]$ if $k$ is large enough. We can therefore satisfy (7.1) for the case where $\omega_{o} \in \mathbb{R}$ by choosing

$$
\hat{\alpha}(s):=\left\{\begin{array}{ll}
\bar{\alpha}(s) & \text { if } 0 \leq s \leq \sigma_{k-1} \\
\gamma_{k}\left(s-\sigma_{k-1}\right) & \text { if } \sigma_{k-1} \leq s<\infty
\end{array} \text { and } \hat{\phi}(s):= \begin{cases}\bar{\phi}(s) & \text { if } 0 \leq s \leq \sigma_{k-1} \\
\lambda_{k}\left(s-\sigma_{k-1}\right) & \text { if } \sigma_{k-1} \leq s<\infty\end{cases}\right.
$$

for $k$ large enough.

Since $\varepsilon$ was arbitrary, we conclude that

$$
w(x) \geq \inf \left\{\int_{0}^{\hat{\tau}(\phi)} \ell(\phi(s), \alpha(s)) \mathrm{d} s+\lambda_{x}(\phi): \alpha \in \mathcal{A}, \phi \in \operatorname{Traj}_{\alpha}(x, f, \partial(\Omega \backslash \mathcal{T}))\right\} .
$$


If $\alpha \in \mathcal{A}$ and $\phi \in \operatorname{Traj}_{\alpha}(x, f, \partial(\Omega \backslash \mathcal{T}))$ are such that $\hat{\tau}(\phi) \neq \tau(\phi)$, then the nonnegativity of $\ell$ and the fact that $w(x)<\omega_{o}$ imply that the associated infimand in (7.9) is

$$
\int_{0}^{\hat{\tau}(\phi)} \ell(\phi(s), \alpha(s)) \mathrm{d} s+1 / 2\left(\omega_{0}+w(x)\right)>w(x),
$$

so such a control is irrelevant for the infimum. This establishes that $w(x) \geq v_{f, \ell, A}(x)$ when $\omega_{o}<\infty$. This proves the proposition. Theorem 6.3 now follows.

I would like to thank Professor H.J. Sussmann for suggesting these problems, and I would like to thank all the members of my doctoral dissertation committee, Professors D.J. Ocone, H.M. Soner, E.D. Sontag, and H.J. Sussmann, for helpful discussions and good advice.

\section{REFERENCES}

[1] O. Alvarez, Bounded-from-below solutions of Hamilton-Jacobi equations. Differential Integral Equations 10 (1997) 419-436.

[2] M. Bardi and I. Capuzzo-Dolcetta, Optimal Control and Viscosity Solutions of Hamilton-Jacobi-Bellman Equations. Birkhäuser, Boston (1997).

[3] M. Bardi and F. Da Lio, On the Bellman equation for some unbounded control problems. NODEA Nonlinear Differential Equations Appl. 4 (1997) 276-285.

[4] M. Bardi, M. Falcone and P. Soravia, Numerical methods for pursuit-evasion games and viscosity solutions, in Stochastic and Differential Games: Theory and Numerical Methods, edited by M. Bardi, T.E.S. Raghavan and T. Parthasarathy. Birkhäuser, Boston (1999).

[5] M. Bardi and P. Soravia, Hamilton-Jacobi equations with singular boundary conditions on a free boundary and applications to differential games. Trans. Amer. Math. Soc. 325 (1991) 205-229.

[6] C. Castaing, Sur les multi-applications mesurables. RAIRO Oper. Res. 1 (1967).

[7] M.G. Crandall, H. Ishii and P.-L. Lions, User's guide to viscosity solutions of second order partial differential equations. Bull. Amer. Math. Soc. 27 (1992) 1-67.

[8] F. Da Lio, On the Bellman equation for infinite horizon problems with unbounded cost functional. Appl. Math. Optim. 41 (1999) 171-197.

[9] W.H. Fleming and H.M. Soner, Controlled Markov Processes and Viscosity Solutions. Springer, New York (1993).

[10] G.B. Folland, Real Analysis: Modern Techniques and their Applications. J. Wiley and Sons, New York (1984).

[11] H. Ishii, On representation of solutions of Hamilton-Jacobi equations with convex Hamiltonians, in Recent Topics in Nonlinear PDE II, edited by K. Masuda and M. Mimura. Kinokuniya Company, Tokyo (1985).

[12] V. Jurdjevic, Geometric Control Theory. Cambridge University Press (1997).

[13] M. Malisoff, A remark on the Bellman equation for optimal control problems with exit times and noncoercing dynamics, in Proc. 38th IEEE Conf. on Decision and Control. Phoenix, AZ (1999) 877-881.

[14] M. Malisoff, Viscosity solutions of the Bellman equation for exit time optimal control problems with vanishing Lagrangians (submitted)

[15] P. Soravia, Pursuit-evasion problems and viscosity solutions of Isaacs equations. SIAM J. Control. Optim. 31 (1993) 604-623.

[16] P. Soravia, Discontinuous viscosity solutions to Dirichlet problems for Hamilton-Jacobi equations with convex Hamiltonians. Comm. Partial Differential Equations 18 (1993) 1493-1514.

[17] P. Soravia, Optimality principles and representation formulas for viscosity solutions of Hamilton-Jacobi equations I: Equations of unbounded and degenerate control problems without uniqueness. Adv. Differential Equations 4 (1999) 275-296.

[18] P. Soravia, Optimal control with discontinuous running cost: Eikonal equation and shape from shading, in Proc. 39th IEEE $C D C$ (to appear).

[19] P. Souganidis, Two-player, zero-sum differential games and viscosity solutions, in Stochastic and Differential Games: Theory and Numerical Methods, edited by M. Bardi, T.E.S. Raghavan and T. Parthasarathy. Birkhäuser, Boston (1999).

[20] H.J. Sussmann, A general theorem on local controllability. SIAM J. Control Optim. 25 (1987) 158-194.

[21] H. Sussmann, From the Brachystochrone problem to the maximum principle, in Proc. of the 35th IEEE Conference on Decision and Control. IEEE Publications, New York (1996) 1588-1594.

[22] H.J. Sussmann, Geometry and optimal control, in Mathematical Control Theory, edited by J. Baillieul and J.C. Willems. Springer-Verlag, New York (1998) 140-198.

[23] H.J. Sussmann and B. Piccoli, Regular synthesis and sufficient conditions for optimality. SISSA Preprint 68/96/M. SIAM J. Control Optim. (to appear).

[24] J. Warga, Optimal Control of Differential and Functional Equations. Academic Press, New York (1972).

[25] M.I. Zelikin and V.F. Borisov, Theory of Chattering Control. Birkhäuser, Boston (1994). 